\title{
Global existence and convergence rates for the smooth solutions to the compressible magnetohydrodynamic equations in the half space
}

\author{
Qing Chen ${ }^{{ }^{*}}$ and Zhong Tan ${ }^{2}$
}

\begin{abstract}
Background: With the characteristics of low pollution and low energy consumption, the magnetohydrodynamics has made widely attention. This paper provides the standard energy method to solve the magnetohydrodynamic equations (MHD) in the half space $\mathbb{R}_{+}^{3}$. It proves the global existence for the compressible (MHD) by combining the careful a priori estimates and the local existence result. This study also considers the large time behaviors of the solutions.

Results: The interactions between the viscous, compressible fluid motion and the magnetic field are modeled by the magnetohydrodynamic system which describes the coupling between the compressible Navier-Stokes equations and the magnetic equations. This study has applied the analytical method to obtain the solutions to (MHD) in $\mathbb{R}_{+}^{3}$. It proves that under the assumption that the initial data are close to the constant state, the global existence of smooth solutions can be established. Moreover, the various decay rates of such solutions in $L^{p}$-norm with $2 \leq p \leq+\infty$ and their derivatives in $L^{2}$-norm can also be derived from combining the decay estimates of the linearized system and the energy method.

Conclusions: This study demonstrates that the global existence and the decay rates for the compressible (MHD) can be established under the similar initial assumptions as for the compressible Navier-Stokes equations. Especially, the results suggest that if the initial velocity is small, the velocity decays at a certain rate. This implies that only under the initial assumption that the data are large, it may reach the requirements of (MHD) power generation, which can be used to achieve the value of industrial application and environmental protection.
\end{abstract}

Keywords: Magnetohydrodynamics; Low pollution; Global existence; Convergence rates

\section{Background}

Magnetohydrodynamics, which combines the environmental fluid mechanics and electrodynamics theories to study the interaction discipline between the conduction fluid and electromagnetic, is the theory of the macroscopic, and it has spanned a very large range of applications (Gerebeau et al. 2006). Due to the lower environmental pollution, especially in energy industry, magnetohydrodynamic power generation is used to conserve

\footnotetext{
*Correspondence: chenqing@xmut.edu.cn

${ }^{1}$ School of Applied Mathematics, Xiamen University of Technology, Ligong Road, 361024 Xiamen, China

Full list of author information is available at the end of the article
}

energy and mitigate pollution in order to protect the environment. In virtue of the industrial importance and theoretical challenges, the study on (MHD) has attracted many scientists. In the present paper, we are interested in the well-posedness theory of (MHD). Many results concerning the existence and uniqueness of (weak, strong or smooth) solutions in one dimension can be found in (Chen and Wang 2002, 2003; Kawashima and Okada 1982) and the references cited therein. In multidimensional case the global existence of weak solutions for the bounded domains has been established recently in (Ducomet and Feireisl 2006; Tan and Wang 2009). The local unique strong solution has been obtained in

\section{黑 Springer}

(c) 2014 Chen and Tan; licensee Springer. This is an Open Access article distributed under the terms of the Creative Commons Attribution License (http://creativecommons.org/licenses/by/4.0), which permits unrestricted use, distribution, and reproduction in any medium, provided the original work is properly credited. 
(Fan and Yu 2009). In (Chen and Tan 2010, 2012) we established the global existence and decay rates of the smooth solutions for the Cauchy problem. However, many fundamental problems for the compressible (MHD) in the half space are still open. In this paper, we will extend our results (Chen and Tan 2010) to the initial boundary problem in the half space.

\section{Results}

In this paper, we will consider the initial boundary value problem for the compressible magnetohydrodynamic equations (MHD) in the half space $\mathbb{R}_{+}^{3}=$ $\left\{x=\left(x^{\prime}, x_{3}\right): x^{\prime} \in \mathbb{R}^{2}, x_{3}>0\right\}$ (cf. (Gerebeau et al. 2006)):

$$
\left\{\begin{array}{l}
\rho_{t}+\operatorname{div}(\rho \mathbf{u})=0, \\
(\rho \mathbf{u})_{t}+\operatorname{div}(\rho \mathbf{u} \otimes \mathbf{u}-\mathbf{P})=\mu_{0} \operatorname{curl} \mathbf{H} \times \mathbf{H}, \\
\mathbf{H}_{t}-\operatorname{curl}(\mathbf{u} \times \mathbf{H})+\frac{1}{\sigma \mu_{0}} \operatorname{curlcurl} \mathbf{H}=0, \operatorname{div} \mathbf{H}=0 .
\end{array}\right.
$$

Here $\rho, \mathbf{u}=\left(u^{1}, u^{2}, u^{3}\right), \mathbf{H}=\left(H^{1}, H^{2}, H^{3}\right)$ represent the density, velocity of the fluid and the magnetic field respectively. $\mu_{0}>0$ stands for permeability of free space, and $\sigma>0$ is the electric conductivity. The stress tensor $\mathbf{P}$ is given by

$$
\mathbf{P}=-p \mathbf{I}+\mu\left(\nabla \mathbf{u}+\nabla \mathbf{u}^{T}\right)+\lambda \operatorname{div} \mathbf{u},
$$

where $p=p(\rho)$ is the pressure and the viscosity coefficients $\lambda$, $\mu$ satisfy

$$
2 \mu+3 \lambda>0 \text { and } \mu>0 .
$$

For convenience, we reformulate the system (1) as

$$
\left\{\begin{array}{l}
\rho_{t}+\operatorname{div}(\rho \mathbf{u})=0, \\
\rho \mathbf{u}_{t}+\rho \mathbf{u} \cdot \nabla \mathbf{u}+\nabla p=\Delta \mathbf{u}+\nabla \operatorname{div} \mathbf{u}+\operatorname{curl} \mathbf{H} \times \mathbf{H}, \\
\mathbf{H}_{t}+\operatorname{curl}(\mathbf{u} \times \mathbf{H})-\Delta \mathbf{H}=0, \operatorname{div} \mathbf{H}=0,
\end{array}\right.
$$

in $(0, \infty) \times \mathbb{R}_{+}^{3}$. Notice that we have normalized some physical constants to be unit but without reducing any essential difficulties for our analysis. We complement (2) the initial condition

$$
(\rho, \mathbf{u}, \mathbf{H})(0, x)=\left(\rho_{0}(x), \mathbf{u}_{0}(x), \mathbf{H}_{0}(x)\right), \quad x \in \mathbb{R}_{+}^{3},
$$

and the following boundary conditions

$$
\left.\mathbf{u}\right|_{\left\{x_{3}=0\right\}}=\mathbf{0},\left.\mathbf{H}\right|_{\left\{x_{3}=0\right\}}=\mathbf{0},
$$

or

$$
\left.\mathbf{u}\right|_{\left\{x_{3}=0\right\}}=\mathbf{0},\left.\mathbf{H} \cdot \mathbf{n}\right|_{\left\{x_{3}=0\right\}}=0, \operatorname{curl} \mathbf{H} \times\left.\mathbf{n}\right|_{\left\{x_{3}=0\right\}}=\mathbf{0},
$$

where $\mathbf{n}=(0,0,-1)$ is the normal vector of $\mathbb{R}_{+}^{3}$. We assume that throughout the paper the initial boundary data satisfy certain compatibility conditions as usual in (Matsumura and Nishida 1983).

Before stating out our results, we shall introduce some standard notations.

Notations. We denote by $L^{p}, W^{m, p}$ the usual Lebesgue and Sobolev spaces on $\mathbb{R}_{+}^{3}$ and $H^{m}=W^{m, 2}$, with norms $|\cdot|_{L^{p}},|\cdot|_{W^{m, p}, \mid}|\cdot|_{H^{m}}$ respectively. For the sake of conciseness, we do not precise in functional space names when they are concerned with scalar-valued or vector-valued functions. We denote $\nabla=\partial_{x}=\left(\partial_{1}, \partial_{2}, \partial_{3}\right)^{t}$, where $\partial_{i}=\partial_{x_{i}}$, and put $\partial_{x}^{l} f=\nabla^{l} f=\nabla\left(\nabla^{l-1} f\right)$ for $l=1,2,3, \cdots$. We assume that $C$ be a positive generic constant throughout this paper that may vary at different places and the integration domain $\mathbb{R}_{+}^{3}$ will be always omitted without any ambiguity. Now our main results can be formulated as the following theorems. Firstly we state the results on the global existence and uniqueness of smooth solutions as:

Theorem 1. Assume that the initial data are close enough to the constant state $(\bar{\rho}, \mathbf{0}, \mathbf{0})$ with $\bar{\rho}>0$, i.e., there exists a constant $\delta_{0}$ such that

$$
\left|\left(\rho_{0}-\bar{\rho}, \mathbf{u}_{0}, \mathbf{H}_{0}\right)\right|_{H^{3}} \leq \delta_{0} .
$$

Then there exists a unique globally smooth solution $(\rho, \mathbf{u}, \mathbf{H})$ of the initial boundary problem (2)-(4) or (2), (3) and (5) such that for any $t \in[0, \infty)$, it holds

$$
\begin{aligned}
& |(\rho-\bar{\rho}, \mathbf{u}, \mathbf{H})(\cdot, t)|_{H^{3}}^{2}+\int_{0}^{t}\left|\partial_{x} \rho(\cdot, s)\right|_{H^{2}}^{2}+\left|\left(\partial_{x} \mathbf{u}, \partial_{x} \mathbf{H}\right)(\cdot, s)\right|_{H^{3}}^{2} d s \\
& \leq C\left|\left(\rho_{0}-\bar{\rho}, \mathbf{u}_{0}, \mathbf{H}_{0}\right)\right|_{H^{3}}^{2} .
\end{aligned}
$$

Remark 1. We will only prove Theorem 1 under the boundary condition (4). Due to the special geometry of the boundary of the half space, a simple calculation shows that the boundary conditions on $\mathbf{H}$ in (5) are equivalent to the following Dirichlet-Neumann boundary conditions:

$$
\left.\partial_{3} H^{1}\right|_{\left\{x_{3}=0\right\}}=\left.\partial_{3} H^{2}\right|_{\left\{x_{3}=0\right\}}=0,\left.\quad H^{3}\right|_{\left\{x_{3}=0\right\}}=0 .
$$

Hence to treat $H^{1}, H^{2}$ as in (Matsumura and Nishida 1983), we can also prove Theorem 1 under the boundary conditions (5) in the similar way as we will proceed.

By imposing some additional conditions on the initial data we will establish the following various decay rates of the solutions obtained in Theorem 1:

Theorem 2. Let $(\rho, \mathbf{u}, \mathbf{H})$ be the solution obtained in Theorem 1 and assume in addition that the initial data $\left(\rho_{0}-\bar{\rho}, \mathbf{u}_{0}, \mathbf{H}_{0}\right) \in L^{1}\left(\mathbb{R}_{+}^{3}\right)$ and there exists $\delta_{1}>0$ such that

$$
\left|\left(\rho_{0}-\bar{\rho}, \mathbf{u}_{0}, \mathbf{H}_{0}\right)\right|_{L^{1}}+\left|\left(\rho_{0}-\bar{\rho}, \mathbf{u}_{0}, \mathbf{H}_{0}\right)\right|_{H^{3}}<\delta_{1},
$$


then for all $t \geq 0$, it holds that

$$
|(\rho-\bar{\rho}, \mathbf{u}, \mathbf{H})(t)|_{L^{p}}=O\left((1+t)^{-\frac{3}{2}\left(1-\frac{1}{p}\right)}\right), \forall p \in[2, \infty],
$$

$$
\left|\partial_{x}(\rho-\bar{\rho}, \mathbf{u}, \mathbf{H})(t)\right|_{L^{2}}=O\left((1+t)^{-\frac{5}{4}}\right),
$$

and

$|(\rho-\bar{\rho}, \mathbf{u}, \mathbf{H})(t)|_{H^{3}} \leq C\left(\delta_{1}\right)\left((1+t)^{-\left(\frac{3}{4}-\varepsilon_{1}\right)}\right), \forall 0<\varepsilon_{1}<\widetilde{\varepsilon}$,

where $\widetilde{\varepsilon}$ is some positive number.

Moreover, if the data satisfy $\left(\rho_{0}-\bar{\rho}, \mathbf{u}_{0}, \mathbf{H}_{0}\right) \in H^{4}\left(\mathbb{R}_{+}^{3}\right)$ and there exists $\delta_{2}>0$ such that

$\left|\left(\rho_{0}-\bar{\rho}, \mathbf{u}_{0}, \mathbf{H}_{0}\right)\right|_{L^{1}}+\left|\left(\rho_{0}-\bar{\rho}, \mathbf{u}_{0}, \mathbf{H}_{0}\right)\right|_{H^{4}}<\delta_{2}$,

then

$\left|\partial_{x}(\rho-\bar{\rho}, \mathbf{u}, \mathbf{H})(t)\right|_{H^{3}} \leq C\left(\delta_{2}\right)(1+t)^{-\left(\frac{5}{4}-\varepsilon_{2}\right)}, \forall 0<\varepsilon_{2}<\widehat{\varepsilon}$,

for all $t \geq 0$, where $\widehat{\varepsilon}<\widetilde{\varepsilon}$ is a positive number. In fact, it holds

| $\left.\left(\partial_{x}^{2} \rho, \partial_{x}^{2} \mathbf{u}, \partial_{x}^{2} \mathbf{H}, \partial_{x}^{3} \mathbf{H}\right)(t)\right|_{L^{2}} \leq C\left(\delta_{2}\right)(1+t)^{-\frac{5}{4}}$,

for all $t \geq 0$.

We will prove the global existence of smooth solutions by the standard energy method in spirit of (Matsumura and Nishida 1983, 1979, 1980). And we remark that we can also obtain the global existence of strong solutions for the small initial data in the $\mathrm{H}^{2}$-framework, which can be proved in the similar way. On the other hand, the $L^{2}-L^{\infty}$ decay rates for the smooth solutions and the $L^{2}$ decay rates for the derivatives of first order are optimal since (9)-(10) concerning ( $\rho-\bar{\rho}, \mathbf{u})$ and $\mathbf{H}$ are the same as the optimal decay rates for the compressible NavierStokes equations (Kagei and Kobayashi 2005) and the heat equation respectively. Related convergence rates of solutions for the Navier-Stokes equations on the unbounded domain can be found in (Kobayashi 2002; Kobayashi and Shibata 1999; Matsumura and Nishida 1979) and the references cited therein. Although our proofs are in spirit of those for the Navier-Stokes equations, (Kagei and Kobayashi 2005; Kobayashi 2002; Kobayashi and Shibata 1999; Matsumura and Nishida 1979, 1980), we should derive the new estimates arising from the presence of the magnetic field and overcome the strong coupling between the mass, momentum equations and magnetic equation. However, it is easy to obtain the optimal decay rates of $\mathbf{H}$ and its first derivatives by the properties of heat kernel.
Indeed, we can rewrite the equation $(19)_{3}$ analogously to the form of $(19)_{1}-(19)_{2}$, i.e.,

$$
\left\{\begin{array}{l}
0_{t}+\operatorname{div} \mathbf{H}=0, \\
\mathbf{H}_{t}+\nabla 0-\Delta \mathbf{H}-\nabla \operatorname{div} \mathbf{H}=S_{3} .
\end{array}\right.
$$

Thus, we can get the estimates of $(0, \mathbf{H})$ which are similar to $(\varrho, \mathbf{v})$ in the system $(19)_{1}-(19)_{2}$. Moreover, we will get the better decay rate of the magnetic field by the elliptic system.

\section{Discussion}

As well known, the heavy emissions of Greenhouse gases, such as $\mathrm{CO}_{2}, \mathrm{CH}_{4}, \mathrm{~N}_{2} \mathrm{O}, \mathrm{SF}_{6}$ cause global warming, and also result in a great deal of harm to the environment. It has been a hot topic and widespread concern to study on how to strictly control the greenhouse gases emissions. In order to profoundly reduce the environment pollution, we must focus on energy structure adjustment. Without any course of mechanical motion, Magnetohydrodynamics (MHD) power generation technology, also called plasma power generation technology, transforms thermal energy and kinetic energy directly into electricity. Thus by applying (MHD) power generation technology, we can realize the desulphuriz and reduce the production of $N O_{x}$ effectively, so as to achieve the effect of high efficiency and low pollution.

To complete the (MHD) generation process, which is of high industrial application value, a conductive gas (plasma) will be directed through a magnetic field with a large velocity, under a high temperature condition. In this situation, how to control the initial velocity of the conductive gas has to be considered. From the results in Section Results, we can conclude that if we assume that the initial data are close enough to the constant state, then there exists a unique globally solution to the (MHD) system and the solution decays at some rates. This indicates that if the initial velocity is sufficiently small, although the solution to the (MHD) system exists globally, then the velocity will decays and never be large, which implies that it may never reach the requirements of (MHD) power generation. However, the problem of the global existence of the solutions with the large initial data is still open.

\section{Conclusions}

In this paper, we demonstrate that the global existence and the decay rates for the compressible (MHD) in $\mathbb{R}_{+}^{3}$ can be established under the similar initial assumptions as for the compressible Navier-Stokes equations which can be seen in (Matsumura and Nishida 1983). It implies that the magnetic field does not affect the decay rates of the velocity. Indeed, the results (9)-(13) in Theorem 2 suggest that the decay rates for the derivatives of the magnetic 
field are the same as the velocity's. And in (14), we cannot get the estimates for $\partial_{x}^{3} \mathbf{u}$ but $\partial_{x}^{3} \mathbf{H}$. Furthermore, the results suggest that if the initial velocity is small, the velocity decays at the optimal rate. This implies that it may never reach the requirements of (MHD) power generation unless giving the gas an large initial velocity.

\section{Methods}

\section{Proof of theorem 1}

In this section, we will prove the existence part of Theorem 1 and the uniqueness is standard so it will be omitted.

\section{Some elementary inequalities}

The first bright idea to reduce many complicated computations lies in that we just need to do the lowest-order and highest-order energy estimates for the solutions. This is motivated by the following observation:

$$
|f|_{H^{k}}^{2} \leq C\left|\left(f, \partial_{x}^{k} f\right)\right|_{L^{2}}^{2}, \forall f \in H^{k}\left(\mathbb{R}_{+}^{3}\right)
$$

The inequality (15) can be easily proved by combing Young's inequality and Gagliardo-Nirenberg's inequality

$$
\left|\partial_{x}^{i} f\right|_{L^{p}} \leq C(p)|f|_{L^{q}}^{\alpha}\left|\partial_{x}^{k} f\right|_{L^{r}}^{(1-\alpha)}, \forall f \in H^{k}\left(\mathbb{R}_{+}^{3}\right)
$$

where $\frac{1}{p}-\frac{i}{3}=\frac{1}{q} \alpha+\left(\frac{1}{r}-\frac{k}{3}\right)(1-\alpha)$ with $i \leq k$. Indeed (16) can be proved by the extension technique together with Gagliardo-Nirenberg's inequality in the whole space. We can obtain the following useful inequality by Hölder inequality and (16):

$$
\begin{gathered}
\left|\partial_{x}^{k}(f g)\right|_{L^{2}} \leq C \\
\forall\left(|f|_{L^{\infty}}\left|\partial_{x}^{k} g\right|_{L^{2}}+\left|\partial_{x}^{k} f\right|_{L^{2}}|g|_{L^{\infty}}\right), \\
\forall f, g \in H^{k}\left(\mathbb{R}_{+}^{3}\right), k \geq 2
\end{gathered}
$$

and the general form of (3) can be deduced directly in the following:

$$
\begin{aligned}
& \left|f_{1} f_{2} \cdots f_{s}\right|_{k} \leq C \sum_{j=1}^{s}\left|f_{1}\right|_{L^{\infty}} \cdots\left|f_{j-1}\right| L_{L^{\infty}}\left|f_{j}\right|_{k}\left|f_{j+1}\right|_{L^{\infty}} \cdots\left|f_{s}\right|_{L^{\infty}}, \\
& \forall f_{j} \in H^{k}\left(\mathbb{R}_{+}^{3}\right), k \geq 2, \text { for } j=1,2, \cdots, s .
\end{aligned}
$$

\section{The linearized system}

We will linearize the problem (2)-(4) as follows. Setting $\gamma=\sqrt{P^{\prime}(\bar{\rho})}, \mu=1 / \bar{\rho}$ and introducing new variables by

$$
\varrho=\rho-\bar{\rho}, \mathbf{v}=\frac{1}{\mu \gamma} \mathbf{u}, \mathbf{H}=\mathbf{H}
$$

Hence the initial boundary value problem (2)-(4) can be reformulated as

$$
\left\{\begin{array}{l}
\varrho_{t}+\gamma \operatorname{divv}=S_{1}, \\
\mathbf{v}_{t}+\gamma \nabla \varrho-\mu \Delta \mathbf{v}-\mu \nabla \operatorname{div} \mathbf{v}=S_{2}, \\
\mathbf{H}_{t}-\Delta \mathbf{H}=S_{3}, \operatorname{div} \mathbf{H}=0, \\
(\varrho, \mathbf{v}, \mathbf{H})(x, 0)=\left(\varrho_{0}, \mathbf{v}_{0}, \mathbf{H}_{0}\right)(x)=\left(\rho_{0}-\bar{\rho}, \mathbf{v}_{0}, \mathbf{H}_{0}\right), \\
\left.\mathbf{v}\right|_{\left\{x_{3}=0\right\}}=\left.\mathbf{H}\right|_{\left\{x_{3}=0\right\}}=\mathbf{0},
\end{array}\right.
$$

where

$$
S_{1}=-\mu \gamma \operatorname{div}(\varrho \mathbf{v})
$$

$$
\begin{aligned}
S_{2}= & \frac{1}{\mu \gamma \rho} \operatorname{curl} \mathbf{H} \times \mathbf{H}+\left(\frac{1}{\rho}-\frac{1}{\bar{\rho}}\right) \Delta \mathbf{v}+\left(\frac{1}{\rho}-\frac{1}{\bar{\rho}}\right) \nabla \operatorname{div} \mathbf{v} \\
& -\mu \gamma \mathbf{v} \cdot \nabla \mathbf{v}-\frac{1}{\mu \gamma}\left[\frac{P^{\prime}(\rho)}{\rho}-\frac{P^{\prime}(\bar{\rho})}{\bar{\rho}}\right] \nabla \varrho,
\end{aligned}
$$

and

$$
S_{3}=-\mu \gamma \operatorname{curl}(\mathbf{v} \times \mathbf{H}) .
$$

In order to state our results more concisely, we define an energy functional as:

$$
\begin{gathered}
N\left(t_{1}, t_{2}\right)=\left\{\sup _{t_{1} \leq t \leq t_{2}}|(\varrho, \mathbf{v}, \mathbf{H})(t)|_{3}^{2}+\int_{t_{1}}^{t_{2}}\left|\partial_{x} \varrho(s)\right|_{2}^{2}\right. \\
\left.+\left|\left(\partial_{x} \mathbf{v}, \partial_{x} \mathbf{H}\right)(s)\right|_{3}^{2} d x\right\}^{\frac{1}{2}},
\end{gathered}
$$

and change the condition of the initial data (6) as

$$
\left|\left(\varrho_{0}, \mathbf{v}_{0}, \mathbf{H}_{0}\right)\right|_{H^{3}} \leq \delta_{0}^{\prime}=\max \left(\frac{1}{2 \mu \gamma}, 1\right) \delta_{0} .
$$

\section{Local and global existence}

We will finish the proof of Theorem 1 in this subsection. First we state out the local existence without proof, since it can be proved in a standard way (Matsumura and Nishida 1980) or can be found in (Ströhmer 1990, Vol'pert and Hudjaev 1972):

Theorem 3. (local existence) Under the assumption (23), there exists a positive constant $T$ such that the initial boundary value problem (19) has a unique solution $(\varrho, \mathbf{v}, \mathbf{H})$ which is continuous in $[0, T] \times \mathbb{R}^{3}$ together with its derivatives of first order in $t$ and of second order in $x$. Moreover, there exists a constant $C_{1}>1$ such that it holds $N(0, t) \leq C_{1} N(0,0)$, for any $t \in[0, T]$.

We will prove in this subsection the following a priori estimate: 
Theorem 4. (a priori estimate) There exists a constant $\delta \ll 1$ such that if $N(0, T) \leq \delta$, then there exists a constant $C_{2}>1$ such that $N(0, T) \leq C_{2} N(0,0)$.

The global existence of smooth solutions will be proved via a continued argument by combining the local existence theorem and the a priori estimate theorem. We shall state the global existence of smooth solutions to the linearized problem (19) as follows.

Proposition 3.1. (global existence) Under the assumptions of Theorem 1, the initial boundary value problem (19) has a unique global solution such that for $t \in[0, \infty)$, it holds $N(0, t) \leq C N(0,0)$. Thus $(\rho, \mathbf{u}, \mathbf{H})$ which satisfies (4) uniquely solves the initial boundary value problem (2)-(4) for all time.

Proof. See in (Chen and Tan 2010).

\section{A priori estimates}

We observe that the a priori assumption in Theorem 4 and the embedding inequality together with the continuity equation (19) 1 imply

$$
\begin{aligned}
& \sup _{0 \leq t \leq T}\left|\left(\varrho, \varrho_{t}, \partial_{x} \varrho, \mathbf{v}, \partial_{x} \mathbf{v}, \mathbf{H}, \partial_{x} \mathbf{H}\right)(t)\right| \\
& \leq C \sup _{0 \leq t \leq T}|(\rho-\bar{\rho}, \mathbf{v}, \mathbf{H})(\cdot, t)|_{H^{3}} \leq C \delta .
\end{aligned}
$$

In particular

$$
\frac{\bar{\rho}}{2} \leq \rho=\varrho+\bar{\rho} \leq 2 \bar{\rho} .
$$

In the sequel, we will always use the smallness assumption of $\delta$ and (24)-(25).

Next we shall do some preparatory work from Lemma 3.1 to Lemma 3.7 . Firstly, we regard the equations $(19)_{2}-(19)_{3}$ as the elliptic system with respect to $x$ variables, i.e.,

$$
\left\{\begin{array}{l}
\Delta \mathbf{v}+\nabla \operatorname{div} \mathbf{v}=\frac{1}{\mu} \mathbf{v}_{t}+\frac{\gamma}{\mu} \nabla \rho-\frac{1}{\mu} S_{2}, \\
\Delta \mathbf{H}=\mathbf{H}_{t}-S_{3}, \\
\left.\mathbf{v}\right|_{\left\{x_{3}=0\right\}}=\mathbf{0},\left.\mathbf{H}\right|_{\left\{x_{3}=0\right\}}=\mathbf{0} .
\end{array}\right.
$$

Thus we have the following estimates which we can found in (Cho et al. 2004):

Lemma 3.1. Under the assumptions of Theorem 4, we have for $k=2,3,4$ that

$$
\left|\partial_{x}^{k} \mathbf{v}\right|_{L^{2}} \leq C\left\{\left|\mathbf{v}_{t}\right|_{k-2}+\left|\partial_{x} \varrho\right|_{k-2}+\left|S_{2}\right|_{k-2}+|\mathbf{v}|_{L^{2}}\right\},
$$

and

$$
\left|\partial_{x}^{k} \mathbf{H}\right|_{L^{2}} \leq C\left\{\left|\mathbf{H}_{t}\right|_{k-2}+\left|S_{3}\right|_{k-2}+\left|\partial_{x} \mathbf{H}\right|_{L^{2}}\right\}
$$

Next we derive the following stokes equation from the equations $(19)_{1}-(19)_{2}$ :

$$
\left\{\begin{array}{l}
\gamma \operatorname{divv}=g=-\frac{d \rho}{d t}-\mu \gamma \varrho \operatorname{div} \mathbf{v}, \\
-\mu \Delta \mathbf{v}+\gamma \nabla \rho=\mathbf{h}=-\mathbf{v}_{t}-\frac{\mu}{\gamma} \nabla g+S_{2}, \\
\left.\mathbf{v}\right|_{\left\{x_{3}=0\right\}}=0 .
\end{array}\right.
$$

We have the following estimates which can be found in (Galdi et al. 1994):

Lemma 3.2. Under the assumptions of Theorem 4, we have for $k=2,3,4$ that

$$
\left|\partial_{x}^{k} \mathbf{v}\right|_{L^{2}}^{2}+\left|\partial_{x}^{k-1} \rho\right|_{L^{2}}^{2} \leq C\left\{|g|_{k-1}^{2}+|h|_{k-2}^{2}\right\} .
$$

Next we shall do the estimates for the terms contained in $N(0, t)$.

Lemma 3.3. Under the assumptions of Theorem 4, we have that

$|(\varrho, \mathbf{v}, \mathbf{H})|_{L^{2}}^{2}+\int_{0}^{t}\left|\partial_{x}(\mathbf{v}, \mathbf{H})\right|_{L^{2}}^{2} d s \leq C N(0,0)^{2}+C \delta N(0, t)^{2}$,

and

$\left|\left(\varrho_{t}, \mathbf{v}_{t}, \mathbf{H}_{t}\right)\right|_{L^{2}}^{2}+\int_{0}^{t}\left|\partial_{x}\left(\mathbf{v}_{t}, \mathbf{H}_{t}\right)\right|_{L^{2}}^{2} d s \leq C N(0,0)^{2}+C \delta N(0, t)^{2}$.

Proof. By multiplying the equations $(19)_{1}-(19)_{3}$ by $\varrho$, $\mathbf{v}$ and $\mathbf{H}$ respectively, integrating over $\mathbb{R}_{+}^{3}$ and adding the resulting equations, we have

$$
\begin{gathered}
\frac{1}{2} \frac{d}{d t}|(\varrho, \mathbf{v}, \mathbf{H})|_{L^{2}}^{2}+\mu\left|\left(\operatorname{div}, \partial_{x} \mathbf{v}\right)\right|_{L^{2}}^{2}+\left|\partial_{x} \mathbf{H}\right|_{L^{2}} \\
=\left\langle S_{1}, \varrho\right\rangle+\left\langle S_{2}, \mathbf{v}\right\rangle+\left\langle S_{3}, \mathbf{H}\right\rangle .
\end{gathered}
$$

We shall estimate the terms in the right-hand side term by term as:

$$
\begin{aligned}
\left\langle S_{1}, \varrho\right\rangle & =\int \gamma \mu(\varrho \mathbf{v}) \cdot \partial_{x} \varrho d x \leq C|\varrho|_{L^{3}}|\mathbf{v}|_{L^{6}}\left|\partial_{x} \varrho\right|_{L^{2}} \\
& \leq C|\varrho|_{H^{1}}\left|\left(\partial_{x} \mathbf{v}, \partial_{x} \varrho\right)\right|_{L^{2}}^{2} \leq C \delta\left|\left(\partial_{x} \mathbf{v}, \partial_{x} \varrho\right)\right|_{L^{2}}^{2} \\
\left\langle S_{2}, \mathbf{v}\right\rangle & \leq C|(\varrho, \mathbf{v}, \mathbf{H})|_{L^{3}}\left|\left(\partial_{x} \varrho, \partial_{x} \mathbf{v}, \partial_{x}^{2} \mathbf{v}, \partial_{x} \mathbf{H}\right)\right|_{L^{2}}|\mathbf{v}|_{L^{6}} \\
& \leq C|(\varrho, \mathbf{v}, \mathbf{H})|_{H^{1}}\left|\left(\partial_{x} \varrho, \partial_{x} \mathbf{v}, \partial_{x}^{2} \mathbf{v}, \partial_{x} \mathbf{H}\right)\right|_{L^{2}}^{2} \\
& \leq C \delta\left|\left(\partial_{x} \varrho, \partial_{x} \mathbf{v}, \partial_{x}^{2} \mathbf{v}, \partial_{x} \mathbf{H}\right)\right|_{L^{2}}^{2} \\
\left\langle S_{3}, \mathbf{H}\right\rangle & \leq C|(\mathbf{v}, \mathbf{H})|_{L^{3}}\left|\left(\partial_{x} \mathbf{v}, \partial_{x} \mathbf{H}\right)\right|_{L^{6}}|\mathbf{H}|_{L^{2}} \\
& \leq C|(\mathbf{v}, \mathbf{H})|_{H^{1}}\left|\left(\partial_{x} \mathbf{v}, \partial_{x} \mathbf{H}\right)\right|_{L^{6}}|\mathbf{H}|_{L^{2}} \\
& \leq C \delta\left|\left(\partial_{x} \mathbf{v}, \partial_{x} \mathbf{H}\right)\right|_{L^{2}}^{2}
\end{aligned}
$$

where Hölder's inequality and Sobolev's inequality are used. Thus by integrating the above inequality in time and the definition of $N(0, t)$, we have got (30). 
Similarly, by taking $\partial_{t}$ to $(19)_{1}-(19)_{3}$, multiplying by $\varrho_{t}, \mathbf{v}_{t}$ and $\mathbf{H}_{t}$ respectively and integrating the resulting inequalities, we obtain

$$
\begin{aligned}
& \left|\left(\varrho_{t}, \boldsymbol{v}_{t}, \boldsymbol{H}_{t}\right)\right|_{L^{2}}^{2}+\int_{0}^{t}\left|\partial_{x}\left(\boldsymbol{v}_{t}, \boldsymbol{H}_{t}\right)\right|_{L^{2}}^{2} d s \\
& \quad \leq C N(0,0)^{2}+C\left|\int_{0}^{t}\left\langle\partial_{t} S_{1}, \varrho_{t}\right\rangle+\left\langle\partial_{t} S_{2}, \boldsymbol{v}_{\boldsymbol{t}}\right\rangle+\left\langle\partial_{t} S_{3}, \boldsymbol{H}_{t}\right\rangle d s\right| .
\end{aligned}
$$

Thus we have to estimate the terms in the right-hand side of the above inequality.

$$
\begin{aligned}
\left\langle\partial_{x} S_{1}, \varrho_{t}\right\rangle & =\int \gamma \mu \partial_{t}(\varrho \operatorname{div} \mathbf{v}+\nabla \varrho \cdot \mathbf{v}) \partial_{t} \varrho d x \\
& \leq C\left|\left(\varrho, \partial_{x} \varrho, \mathbf{v}, \partial_{x} \mathbf{v}\right)\right|_{L^{3}}\left|\left(\varrho_{t}, \partial_{x} \varrho_{t}, \mathbf{v}_{t}, \partial_{x} \mathbf{v}_{t}\right)\right|_{L^{2}}\left|\varrho_{t}\right|_{L^{6}} \\
& \leq C\left|\left(\varrho, \partial_{x} \varrho, \mathbf{v}, \partial_{x} \mathbf{v}\right)\right|_{H^{1}}\left|\left(\varrho_{t}, \partial_{x} \varrho_{t}, \mathbf{v}_{t}, \partial_{x} \mathbf{v}_{t}\right)\right|_{L^{2}}^{2} \\
& \leq C \delta\left|\left(\partial_{x} \varrho, \partial_{x}^{2} \varrho, \partial_{x} \mathbf{v}, \partial_{x}^{2} \mathbf{v}, \partial_{x}^{3} \mathbf{v}, \partial_{x} \mathbf{H}, \partial_{x}^{2} \mathbf{H}\right)\right|_{L^{2}}^{2} \\
& \leq C \delta N(0, t)^{2}
\end{aligned}
$$

where we can get the $L^{2}$-norm estimates of $\varrho_{t}, \partial_{x} \varrho_{t}, \mathbf{v}_{t}$, and $\partial_{x} \mathbf{v}_{t}$ with the aid of the equation $(19)_{1}-(19)_{2}$ and (24). Similarly we have that

$$
\begin{aligned}
\left\langle\partial_{t} S_{2}, \mathbf{v}_{t}\right\rangle \leq & C\left|\left\langle\partial_{t}(\varrho \operatorname{curl} \mathbf{H} \times \mathbf{H}, \varrho \Delta \mathbf{v}, \varrho \nabla \operatorname{div} \mathbf{v}, \mathbf{v} \cdot \nabla \mathbf{v}, \varrho \cdot \nabla \varrho), \mathbf{v}_{t}\right\rangle\right| \\
\leq & C\left|\left(\varrho, \varrho t, \mathbf{v}, \partial_{x} \mathbf{v}, \mathbf{H}, \partial_{x} \mathbf{H}\right)\right|_{L^{3}} \\
& \times\left|\left(\partial_{x} \varrho, \partial_{x} \varrho_{t}, \mathbf{v}_{t}, \partial_{x}^{2} \mathbf{v}, \partial_{x} \mathbf{v}_{t}, \partial_{x}^{2} \mathbf{v}_{t}, \partial_{x} \mathbf{H}, \mathbf{H}_{t}, \partial_{x} \mathbf{H}_{t}\right)\right|_{L^{2}}\left|\mathbf{v}_{t}\right|_{L^{6}} \\
\leq & C\left|\left(\varrho, \partial_{x} \varrho, \mathbf{v}, \partial_{x} \mathbf{v}, \mathbf{H}, \partial_{x} \mathbf{H}\right)\right|_{H^{1}} \\
& \times\left|\left(\partial_{x} \varrho, \partial_{x}^{2} \varrho, \partial_{x}^{3} \varrho, \partial_{x} \mathbf{v}, \partial_{x}^{2} \mathbf{v}, \partial_{x}^{3} \mathbf{v}, \partial_{x}^{4} \mathbf{v}, \partial_{x} \mathbf{H}, \partial_{x}^{2} \mathbf{H}, \partial_{x}^{3} \mathbf{H}\right)\right|_{L^{2}}^{2} \\
\leq & C \delta\left|\left(\partial_{x} \varrho, \partial_{x}^{2} \varrho, \partial_{x}^{3} \varrho, \partial_{x} \mathbf{v}, \partial_{x}^{2} \mathbf{v}, \partial_{x}^{3} \mathbf{v}, \partial_{x}^{4} \mathbf{v}, \partial_{x} \mathbf{H}, \partial_{x}^{2} \mathbf{H}, \partial_{x}^{3} \mathbf{H}\right)\right|_{L^{2}}^{2}
\end{aligned}
$$

and

$$
\begin{aligned}
\left\langle\partial_{t} S_{3}, \mathbf{H}_{t}\right\rangle & \leq C\left|\left\langle\partial_{t} \operatorname{curl}(\mathbf{v} \times \mathbf{H}), \mathbf{H}_{t}\right\rangle\right| \\
& \leq C\left|\left(\mathbf{v}, \partial_{x} \mathbf{v}, \mathbf{H}, \partial_{x} \mathbf{H}\right)\right|_{L^{3}}\left|\left(\mathbf{v}_{t}, \partial_{x} \mathbf{v}_{t}, \mathbf{H}_{t}, \partial_{x} \mathbf{H}_{t}\right)\right|_{L^{2}}\left|\mathbf{H}_{t}\right|_{L^{6}} \\
& \leq C|(\mathbf{v}, \mathbf{H})|_{H^{2}}\left|\left(\partial_{x} \varrho, \partial_{x}^{2} \varrho, \partial_{x} \mathbf{v}, \partial_{x}^{2} \mathbf{v}, \partial_{x}^{3} \mathbf{v}, \partial_{x} \mathbf{H}, \partial_{x}^{2} \mathbf{H}, \partial_{x}^{3} \mathbf{H}\right)\right|_{L^{2}}^{2} \\
& \leq C \delta\left|\left(\partial_{x} \varrho, \partial_{x}^{2} \varrho, \partial_{x} \mathbf{v}, \partial_{x}^{2} \mathbf{v}, \partial_{x}^{3} \mathbf{v}, \partial_{x} \mathbf{H}, \partial_{x}^{2} \mathbf{H}, \partial_{x}^{3} \mathbf{H}\right)\right|_{L^{2}}^{2} .
\end{aligned}
$$

Together with these inequalities, we can deduce the inequality (31). Hence the proof of Lemma 3.3 is complete.

Next we estimate the $L^{2}$-norm of the first derivatives of $\mathbf{v}$ and $\mathbf{H}$.

Lemma 3.4. Under the assumptions of Theorem 4, we have

$$
\begin{aligned}
\left|\partial_{x}(\mathbf{v}, \mathbf{H})\right|_{L^{2}}^{2} & +\int_{0}^{t}\left|\left(\varrho_{t}, \mathbf{v}_{t}, \mathbf{H}_{t}\right)(s)\right|_{L^{2}}^{2} d s \leq C N(0,0)^{2} \\
& +C \delta N(0, t)^{2},
\end{aligned}
$$

and

$$
\begin{aligned}
\left|\partial_{x}\left(\mathbf{v}_{t}, \mathbf{H}_{t}\right)\right|_{L^{2}}^{2} & +\int_{0}^{t}\left|\left(\varrho_{t t}, \mathbf{v}_{t t}, \mathbf{H}_{t t}\right)(s)\right|_{L^{2}}^{2} d s \leq C N(0,0)^{2} \\
& +C \delta N(0, t)^{2}
\end{aligned}
$$

Proof. By multiplying $\varrho_{t}, \mathbf{v}_{t}$ and $\mathbf{H}_{t}$ to the equations $(19)_{1}-(19)_{3}$ respectively, integrating over $\mathbb{R}_{+}^{3}$ and adding the resulting equations, we have

$$
\begin{aligned}
\frac{d}{d t} \int & \left(\frac{\mu}{2}\left|\partial_{x} \mathbf{v}\right|^{2}+\frac{\mu}{2}|\operatorname{div} \mathbf{v}|^{2}+\frac{1}{2}\left|\partial_{x} \mathbf{H}\right|^{2}-\gamma \varrho \operatorname{div} \mathbf{v}\right) d x \\
& +\int\left(\varrho_{t}^{2}+\left|\mathbf{v}_{t}\right|^{2}+\left|\mathbf{H}_{t}\right|^{2}+2 \gamma \varrho_{t} \operatorname{divv}\right) d x \\
= & \int\left\langle S_{1}, \varrho_{t}\right\rangle+\left\langle S_{2}, \mathbf{v}_{t}\right\rangle+\left\langle S_{3}, \mathbf{H}_{t}\right\rangle d x \\
\leq & \epsilon\left(\left|\varrho_{t}\right|_{L^{2}}^{2}+\left|\mathbf{v}_{t}\right|_{L^{2}}^{2}+\left|\mathbf{H}_{t}\right|_{L^{2}}^{2}\right)+C(\epsilon)\left(\left|S_{1}\right|_{L^{2}}^{2}+\left|S_{2}\right|_{L^{2}}^{2}+\left|S_{3}\right|_{L^{2}}^{2}\right) .
\end{aligned}
$$

Since

$$
\begin{aligned}
\left|S_{1}\right|_{L^{2}}^{2} & \leq C|(\varrho, \mathbf{v})|_{L^{\infty}}\left|\partial_{x}(\varrho, \mathbf{v})\right|_{L^{2}}^{2} \leq C \delta\left|\partial_{x}(\varrho, \mathbf{v})\right|_{L^{2}}^{2}, \\
\left|S_{2}\right|_{L^{2}}^{2} & \leq C|(\varrho, \mathbf{v}, \mathbf{H})|_{L^{\infty}}\left|\left(\partial_{x} \varrho, \partial_{x} \mathbf{v}, \partial_{x}^{2} \mathbf{v}, \partial_{x} \mathbf{H}\right)\right|_{L^{2}}^{2} \\
& \leq C \delta\left|\left(\partial_{x} \varrho, \partial_{x} \mathbf{v}, \partial_{x}^{2} \mathbf{v}, \partial_{x} \mathbf{H}\right)\right|_{L^{2}}^{2},
\end{aligned}
$$

and

$$
\left|S_{3}\right|_{L^{2}}^{2} \leq C|(\mathbf{v}, \mathbf{H})|_{L^{\infty}}\left|\partial_{x}(\mathbf{v}, \mathbf{H})\right|_{L^{2}}^{2} \leq C \delta\left|\partial_{x}(\mathbf{v}, \mathbf{H})\right|_{L^{2}}^{2},
$$

thus by integrating (34) in time and with the aid of the smallness of $\epsilon$ and Cauchy's inequality, we obtain

$$
\begin{aligned}
& \left|\partial_{x}(\mathbf{v}, \mathbf{H})\right|_{L^{2}}^{2}+\int_{0}^{t}\left|\left(\varrho_{t}, \mathbf{v}_{t}, \mathbf{H}_{t}\right)(s)\right|_{L^{2}}^{2} d s \\
& \quad \leq C N(0,0)^{2}+C \delta N(0, t)^{2}+C\left(|\varrho|_{L^{2}}^{2}+\int_{0}^{t}\left|\partial_{x} \mathbf{v}(s)\right|_{L^{2}}^{2} d s\right)
\end{aligned}
$$

which together with (30) yields (32). And we can also get (33) in the similar way. The proof of Lemma 3.4 is complete.

Next we estimate the $L^{2}$-Norms of the first derivatives of $\varrho$. We shall divide the estimates into two parts. Firstly we denote the tangential derivatives by $\partial=\left(\partial_{1}, \partial_{2}\right)$. And it is easy to see that the tangential derivatives of the solution of (19) satisfy the same boundary conditions in (19).

By taking $\partial$ to the equations $(19)_{1}-(19)_{2}$ and multiplying them with $\partial \varrho$ and $\partial \mathbf{v}$ respectively, we can deduce that

$$
\begin{aligned}
& \frac{1}{2} \frac{d}{d t} \int|\partial \varrho|^{2}+|\partial \mathbf{v}|^{2} d x+\int \mu\left|\partial \partial_{x} \mathbf{v}\right|^{2}+\mu|\partial \operatorname{div} \mathbf{v}|^{2} d x \\
& \quad=\left\langle\partial S_{1}, \partial \varrho\right\rangle+\left\langle\partial S_{2}, \partial \mathbf{v}\right\rangle
\end{aligned}
$$

Define the material derivative $\frac{d \rho}{d t}=\rho_{t}+\nabla \rho \cdot \mathbf{u}$. Then by the continuity equation (19) ${ }_{1}$ and the formula of variable substitution (18), we have

$$
\frac{D \varrho}{D t}=-\gamma \operatorname{div} \mathbf{v}-\mu \gamma \varrho \operatorname{div} \mathbf{v}
$$


where $\frac{D \varrho}{D t}=\frac{d \rho}{d t}=\varrho_{t}+\mu \gamma \nabla \varrho \cdot \mathbf{v}$. By taking $\partial$ to the equation (36), we can obtain the following inequality:

$$
\left|\partial\left(\frac{D \varrho}{D t}\right)\right|_{L^{2}}^{2} \leq C\left(\left.\partial \partial_{x} \mathbf{v}\right|_{L^{2}} ^{2}+|\partial(\varrho \operatorname{div} \mathbf{v})|_{L^{2}}^{2}\right)
$$

then by multiplying a small enough constant $\alpha$ to it, together with (35), and integrating in time, we have

$$
\begin{aligned}
& |\partial(\varrho, \mathbf{v})|_{L^{2}}^{2}+\int_{0}^{t}\left|\partial\left(\frac{D \varrho}{D t}, \partial_{x} \mathbf{v}\right)\right|_{L^{2}}^{2} d s \\
& \leq C N(0,0)^{2}+C\left|\int_{0}^{t}\left\langle\partial S_{1}, \partial \varrho\right\rangle+\left\langle\partial S_{2}, \partial \mathbf{v}\right\rangle d s\right| \\
& \quad+C \int_{0}^{t}|\partial(\varrho \operatorname{div} \mathbf{v})|_{L^{2}}^{2} d s \\
& \leq C N(0,0)^{2}+C N(0, t)^{2}+C\left|\int_{0}^{t}\left\langle\partial S_{1}, \partial \varrho\right\rangle+\left\langle\partial S_{2}, \partial \mathbf{v}\right\rangle d s\right| .
\end{aligned}
$$

Similarly, we can obtain the following lemma and we omit the proof of it.

Lemma 3.5. Under the assumptions of Theorem 4, we have for $\mathrm{k}=1,2,3$ that

$$
\begin{aligned}
& \left|\partial^{k}(\varrho, \mathbf{v})\right|_{L^{2}}^{2}+\int_{0}^{t}\left|\partial^{k}\left(\frac{D \varrho}{D t}, \partial_{x} \mathbf{v}\right)\right|_{L^{2}}^{2} d s \\
& \leq C N(0,0)^{2}+C N(0, t)^{2}+C \mid \int_{0}^{t}\left\langle\partial^{k} S_{1}, \partial^{k} \varrho\right\rangle \\
& \quad+\left\langle\partial^{k} S_{2}, \partial^{k} \mathbf{v}\right\rangle d s \mid .
\end{aligned}
$$

Next we have to obtain the estimates for the normal derivatives of solution. We can derive the following equations from $(19)_{1}-(19)_{2}$.

$$
\begin{aligned}
& \partial_{3} \varrho_{t}+\gamma \partial_{3} \operatorname{div} \mathbf{v}=-\mu \gamma \partial_{3}(\varrho \operatorname{div} \mathbf{v})-\mu \gamma \partial_{3}(\nabla \varrho \cdot \mathbf{v}), \\
& \mathbf{v}_{t}^{3}+\gamma \partial_{3} \varrho-\mu \Delta \mathbf{v}^{3}-\mu \partial_{3} \operatorname{div} \mathbf{v}=S_{2}^{3}
\end{aligned}
$$

where $S_{2}=\left(S_{2}^{1}, S_{2}^{2}, S_{2}^{3}\right)^{T}$. To eliminate the term $\partial_{33} \mathbf{v}^{3}$, we have the following equality from the above equalities

$$
\begin{aligned}
\frac{2 \mu}{\gamma} \partial_{3} \varrho_{t}+\gamma \partial_{3} \varrho= & -\mathbf{v}_{t}^{3}+\mu\left(\partial_{11} \mathbf{v}^{3}+\partial_{22} \mathbf{v}^{3}+\partial_{13} \mathbf{v}^{1}+\partial_{23} \mathbf{v}^{2}\right) \\
& -2 \mu^{2} \partial_{3}(\varrho \operatorname{div} \mathbf{v})-2 \mu^{2} \partial_{3}(\nabla \varrho \cdot \mathbf{v})+S_{2}^{3}
\end{aligned}
$$

And we can also derive the following equality from (36)

$$
\begin{aligned}
\frac{2 \mu}{\gamma} \partial_{3}\left(\frac{D \varrho}{D t}\right)+\gamma \partial_{3} \varrho= & -\mathbf{v}_{t}^{3}+\mu\left(\partial_{11} \mathbf{v}^{3}+\partial_{22} \mathbf{v}^{3}+\partial_{13} \mathbf{v}^{1}+\partial_{23} \mathbf{v}^{2}\right) \\
& -2 \mu^{2} \partial_{3}(\varrho \text { divv })+S_{2}^{3} .
\end{aligned}
$$

By multiplying (38) with $\partial_{3} \varrho$ and integrating on $\mathbb{R}_{+}^{3}$, we have

$$
\begin{aligned}
& \frac{\mu}{\gamma} \frac{d}{d t}\left|\partial_{3} \varrho\right|_{L^{2}}^{2}+\gamma\left|\partial_{3} \varrho\right|_{L^{2}}^{2} \\
& =\int\left\{-\mathbf{v}_{t}^{3}+\mu\left(\partial_{11} \mathbf{v}^{3}+\partial_{22} \mathbf{v}^{3}+\partial_{13} \mathbf{v}^{1}+\partial_{23} \mathbf{v}^{2}\right)\right. \\
& \left.\quad-2 \mu^{2} \partial_{3}(\varrho \operatorname{div} \mathbf{v})-2 \mu^{2} \partial_{3}(\nabla \varrho \cdot \mathbf{v})+S_{2}^{3}\right\} \partial_{3} \varrho d x \\
& \leq \frac{\gamma}{2}\left|\partial_{3} \varrho\right|_{L^{2}}^{2}+C(\gamma)\left|\left(\mathbf{v}_{t}, \partial \partial_{x} \mathbf{v}, \partial_{3}(\varrho \operatorname{div} \mathbf{v}), S_{2}\right)\right|_{L^{2}}^{2} \\
& \quad+C\left|\int \partial_{3}(\nabla \varrho \cdot \mathbf{v}) \partial_{3} \varrho d x\right|,
\end{aligned}
$$

then integrating this in time, it implies

$$
\begin{aligned}
\left|\partial_{3} \varrho\right|_{L^{2}}^{2} & +\int_{0}^{t}\left|\partial_{3} \varrho(s)\right|_{L^{2}}^{2} d s \\
\leq & C N(0,0)^{2}+C \int_{0}^{t}\left|\left(\mathbf{v}_{t}, \partial \partial_{x} \mathbf{v}, \partial_{3}(\varrho \operatorname{div} \mathbf{v}), S_{2}\right)\right|_{L^{2}}^{2} \\
& +\left|\int \partial_{3}(\nabla \varrho \cdot \mathbf{v}) \partial_{3} \varrho d x\right| d s \\
\leq & C N(0,0)^{2}+C \int_{0}^{t}\left|\left(\mathbf{v}_{t}, \partial \partial_{x} \mathbf{v}\right)\right|_{L^{2}}^{2} d s+C \delta N(0, t)^{2} .
\end{aligned}
$$

Similarly, by multiplying $\partial_{3}\left(\frac{D \varrho}{D t}\right)$ to (39) and integrating it, we have

$$
\begin{aligned}
& \left|\partial_{3} \varrho\right|_{L^{2}}^{2}+\int_{0}^{t}\left|\partial_{3}\left(\frac{D \varrho}{D t}\right)(s)\right|_{L^{2}}^{2} d s \\
& \leq C N(0,0)^{2}+C \int_{0}^{t}\left|\left(\mathbf{v}_{t}, \partial \partial_{x} \mathbf{v}, \partial_{3}(\varrho \operatorname{div} \mathbf{v}), S_{2}\right)\right|_{L^{2}}^{2} \\
& \leq C N(0,0)^{2}+C \delta N(0, t)^{2}+C \int_{0}^{t}\left|\left(\mathbf{v}_{t}, \partial \partial_{x} \mathbf{v}\right)\right|_{L^{2}}^{2}
\end{aligned}
$$

This together with (41) yields

$$
\begin{aligned}
& \left|\partial_{3} \varrho\right|_{L^{2}}^{2}+\int\left|\partial_{3} \varrho(s)\right|_{L^{2}}^{2}+\left|\partial_{3}\left(\frac{D \varrho}{D t}\right)(s)\right|_{L^{2}}^{2} d s \\
& \quad \leq C N(0,0)^{2}+C \delta N(0, t)^{2}+C \int_{0}^{t}\left|\left(\mathbf{v}_{t}, \partial \partial_{x} \mathbf{v}\right)\right|_{L^{2}}^{2} d s
\end{aligned}
$$

Similarly, by taking $\partial^{k} \partial_{3}^{l}$ to (38) and (39), multiplying with $\partial^{k} \partial_{3}^{1+l} \varrho$ and $\partial^{k} \partial_{3}^{1+l}\left(\frac{D \varrho}{D t}\right)$ respectively, we will get the general form of (42) as follows: 
Lemma 3.6. Under the assumptions of Theorem 4, we have for $k+l=0,1,2$ that

$$
\begin{aligned}
& \left|\partial^{k} \partial_{3}^{1+l} \varrho\right|_{L^{2}}^{2}+\int_{0}^{t}\left|\partial^{k} \partial_{3}^{1+l} \varrho\right|_{L^{2}}^{2}+\left|\partial^{k} \partial_{3}^{1+l}\left(\frac{D \varrho}{D t}\right)(s)\right|_{L^{2}}^{2} d s \\
& \leq C N(0,0)^{2}+C \delta N(0, t)^{2}+C \int_{0}^{t}\left|\partial^{k} \partial_{3}^{l}\left(\mathbf{v}_{t}, \partial \partial_{x} \mathbf{v}\right)\right|_{L^{2}}^{2} d s .
\end{aligned}
$$

Proof. Here we only to prove the case when $k+l=2$. As in (42), we can obtain

$$
\begin{aligned}
& \left|\partial^{k} \partial_{3}^{1+l} \varrho\right|_{L^{2}}^{2}+\int_{0}^{t}\left|\partial^{k} \partial_{3}^{1+l} \varrho\right|_{L^{2}}^{2}+\left|\partial^{k} \partial_{3}^{1+l}\left(\frac{D \varrho}{D t}\right)(s)\right|_{L^{2}}^{2} d s \\
& \leq C N(0,0)^{2}+C \int_{0}^{t}\left|\partial^{k} \partial_{3}^{l}\left(\mathbf{v}_{t}, \partial \partial_{x} \mathbf{v}, \partial_{3}(\varrho \operatorname{div} \mathbf{v}), S_{2}\right)\right|_{L^{2}}^{2} \\
& \quad+\left|\int \partial^{k} \partial_{3}^{1+l}(\nabla \varrho \cdot \mathbf{v}) \cdot \partial^{k} \partial_{3}^{1+l} \varrho d x\right| d s .
\end{aligned}
$$

By (17), we have

$$
\begin{aligned}
\int_{0}^{t}\left|\partial_{x}^{3}(\varrho \operatorname{div} \mathbf{v})\right|_{L^{2}}^{2} d s & \leq C \int_{0}^{t}|\varrho, \operatorname{div} \mathbf{v}|_{L^{\infty}}^{2}\left|\partial_{x}^{3} \varrho, \partial_{x}^{3} \operatorname{div} \mathbf{v}\right|_{L^{2}}^{2} d s \\
& \leq C \delta N(0, t)^{2} .
\end{aligned}
$$

Similarly,

$$
\begin{aligned}
\int_{0}^{t}\left|\partial_{x}^{2} S_{2}\right|_{L^{2}}^{2} d s \leq & C \int_{0}^{t}|\varrho, \mathbf{v}, \mathbf{H}|_{L^{\infty}}^{2}\left|\partial_{x}^{3} \varrho, \partial_{x}^{3} \mathbf{v}, \mathbf{H}\right|_{L^{2}}^{2} d s \\
& +C \int_{0}^{t}\left|\varrho, \partial_{x} \varrho\right|_{L^{\infty}}^{2}\left|\partial_{x}^{2} \mathbf{v}\right|_{1}^{2}+\left|\partial_{x}^{2} \varrho\right|_{L^{4}}^{2}\left|\partial_{x}^{2} \mathbf{v}\right|_{L^{4}}^{2} d s \\
\leq & C \delta N(0, t)^{2}+C \int_{0}^{t}\left|\partial_{x}^{2} \varrho\right|_{1}^{2}\left|\partial_{x}^{2} \mathbf{v}\right|_{1}^{2} d s \\
\leq & C \delta N(0, t)^{2} .
\end{aligned}
$$

Moreover, by using the identity

$$
\left(\partial^{k} \partial_{3}^{1+l} \nabla \varrho \cdot \mathbf{v}\right) \cdot \partial^{k} \partial_{3}^{1+l} \varrho=\nabla \frac{\left|\partial^{k} \partial_{3}^{1+l} \varrho\right|^{2}}{2} \cdot \mathbf{v}
$$

and integration by parts, we shall obtain

$$
\int_{0}^{t} \int \partial^{k} \partial_{3}^{1+l}(\nabla \varrho \cdot \mathbf{v}) \cdot \partial^{k} \partial_{3}^{1+l} \varrho d x d s \leq C \delta N(0, t)^{2} .
$$

Substituting these inequalities into (44), we get (43).

Last by taking $\partial_{x}^{l} \partial^{k}$ to (28), and by Lemma 3.2, we have
Lemma 3.7. Under the assumptions of Theorem 4, we have for $k+l=0,1,2$ that

$$
\begin{aligned}
\int_{0}^{t}\left|\partial_{x}^{2+l} \partial^{k} \mathbf{v}\right|_{L^{2}} & +\left|\partial_{x}^{1+l} \partial^{k} \varrho\right|_{L^{2}} d s \leq C \int_{0}^{t}\left|\partial^{k} \mathbf{v}_{t}\right|_{l} \\
& +\left|\partial^{k}\left(\frac{D \varrho}{D t}\right)\right|_{1+l} d s+C \delta N(0, t)^{2}
\end{aligned}
$$

Now we will finish the proof of Theorem 4 by doing the estimates for the lowest-order and highest-order derivatives. In the sequel, we divide the a priori estimates into three parts.

Part 1: estimates for the lowest derivatives of $\varrho, \boldsymbol{v}, \boldsymbol{H}$

Proposition 3.2. Under the assumptions of Theorem 4, we have

$$
\begin{aligned}
|(\varrho, \mathbf{v}, \mathbf{H})|_{L^{2}}^{2} & +\int_{0}^{t}\left|\partial_{\mathcal{X}}(\varrho, \mathbf{v}, \mathbf{H})(s)\right|_{L^{2}}^{2} d s \leq C N(0,0)^{2} \\
& +C \delta N(0, t)^{2} .
\end{aligned}
$$

Proof. Due to (30), we have only to estimate $\int_{0}^{t}\left|\partial_{x} \varrho(s)\right|_{L^{2}}^{2} d s$. First by Lemma $3.5, k=0,1$, we have

$$
\begin{aligned}
& \int_{0}^{t}\left|\frac{D \varrho}{D t}(s)\right|_{L^{2}}^{2}+\left|\partial\left(\frac{D \varrho}{D t}\right)(s)\right|_{L^{2}}^{2}+\left|\partial \partial_{x} \mathbf{v}\right|_{L^{2}}^{2} d s \\
& \quad \leq C N(0,0)^{2}+C \delta N(0, t)^{2}+C \delta \int_{0}^{t}\left|\left(S_{1}, S_{2}, S_{3}\right)(s)\right|_{L^{2}}^{2} d s \\
& \quad \leq C N(0,0)^{2}+C \delta N(0, t)^{2} .
\end{aligned}
$$

And by (42), we have

$$
\begin{aligned}
\int_{0}^{t}\left|\partial_{3}\left(\frac{D \varrho}{D t}\right)\right|_{L^{2}}^{2} d s \leq & C N(0,0)^{2}+C \int_{0}^{t}\left|\left(\mathbf{v}_{t}, \partial \partial_{x} \mathbf{v}\right)\right|_{L^{2}}^{2} d s \\
& +C \delta N(0, t)^{2}
\end{aligned}
$$

Thus (47)-(48) together with (32) implies

$$
\int_{0}^{t}\left|\frac{D \varrho}{D t}(s)\right|_{1}^{2} d s \leq C N(0,0)^{2}+C \delta N(0, t)^{2} .
$$

By Lemma 3.4, $k=l=0$, and integrating in time, we obtain

$$
\int_{0}^{t}\left|\partial_{x} \varrho\right|_{L^{2}}^{2} d s \leq C \int_{0}^{t}\left|\mathbf{v}_{t}\right|_{L^{2}}^{2}+\mid\left(\left.\frac{D \varrho}{D t}\right|_{1} ^{2}\right) d s+C \delta N(0, t)^{2}
$$

This combining with (49) and (32), yields the estimate for $\int_{0}^{t}\left|\partial_{x} \varrho(s)\right|_{L^{2}}^{2} d s$. 
Part 2: estimates for the highest derivatives of $\varrho, \boldsymbol{v}, \boldsymbol{H}$

Proposition 3.3. Under the assumptions of Theorem 4, we have

$$
\begin{aligned}
\left|\partial_{x}^{3}(\varrho, \mathbf{v}, \mathbf{H})\right|_{L^{2}}^{2} & +\int_{0}^{t}\left|\left(\partial_{x}^{3} \varrho, \partial_{x}^{4} \mathbf{v}, \partial_{x}^{4} \mathbf{H}\right)(s)\right|_{L^{2}}^{2} d s \leq C N(0,0)^{2} \\
& +C \delta N(0, t)^{2} .
\end{aligned}
$$

Proof. We divide the proof into three steps as follows.

i) By Lemma 2.8, $k=3$ and Lemma $2.9, k=2, l=0$, we have

$$
\begin{gathered}
\left|\partial^{2} \partial_{x} \varrho\right|_{L^{2}}^{2}+\int_{0}^{t}\left|\left(\partial^{2} \partial_{3} \varrho, \partial^{2} \partial_{x}\left(\frac{D \varrho}{D t}\right), \partial^{3} \partial_{x} \mathbf{v}\right)\right|_{L^{2}}^{2} d s \\
\leq C N(0,0)^{2}+C \delta N(0, t)^{2}+C \mid \int_{0}^{t}\left\langle\partial^{k} S_{1}, \partial^{k} \varrho\right\rangle \\
+\left.\left\langle\partial^{k} S_{2}, \partial^{k} \mathbf{v}\right\rangle d s\left|+C \int_{0}^{t}\right| \partial^{2} \mathbf{v}_{t}\right|_{L^{2}} ^{2} \\
\leq C N(0,0)^{2}+C \delta N(0, t)^{2}+C \int_{0}^{t}\left|\partial^{2} \mathbf{v}_{t}\right|_{L^{2}}^{2} d s,
\end{gathered}
$$

where we get the last inequality as in the proof of Lemma 3.6. By Lemma 3.7, $k=2, l=0$, we have

$$
\begin{gathered}
\int_{0}^{t}\left|\left(\partial_{x}^{2} \partial^{2} \mathbf{v}, \partial_{x} \partial^{2} \varrho\right)\right|_{L^{2}}^{2} d s \leq C \int_{0}^{t} \mid\left(\partial^{2} \mathbf{v}_{t},\left.\partial^{2} \partial_{x}\left(\frac{D \varrho}{D t}\right)\right|_{L^{2}} ^{2} d s\right. \\
+C \delta N(0, t)^{2} .
\end{gathered}
$$

This together with (51) yields

$$
\begin{array}{r}
\left|\partial^{2} \partial_{x} \varrho\right|_{L^{2}}^{2}+\int_{0}^{t}\left|\left(\partial^{2} \partial_{x} \varrho, \partial^{2} \partial_{x}\left(\frac{D \varrho}{D t}\right), \partial^{2} \partial_{x}^{2} \mathbf{v}\right)\right|_{L^{2}}^{2} d s \\
\leq C N(0,0)^{2}+C \delta N(0, t)^{2}+C \int_{0}^{t}\left|\partial^{2} \mathbf{v}_{t}\right|_{L^{2}}^{2} d s .
\end{array}
$$

By Lemma 3.6, $k=l=1$, and (52), we have

$$
\begin{gathered}
\left|\partial \partial_{x}^{2} \varrho\right|_{L^{2}}^{2}+\int_{0}^{t}\left|\left(\partial \partial_{x}^{2} \varrho, \partial \partial_{x}^{2}\left(\frac{D \varrho}{D t}\right), \partial^{2} \partial_{x}^{2} \mathbf{v}\right)\right|_{L^{2}}^{2} d s \\
\leq C N(0,0)^{2}+C \delta N(0, t)^{2}+C \int_{0}^{t}\left|\partial_{x}^{2} \mathbf{v}_{t}\right|_{L^{2}}^{2} d s .
\end{gathered}
$$

Then by Lemma 3.7, $k=l=1$, and with the help of (15), (31) and (47), we have

$$
\begin{aligned}
\int_{0}^{t} & \left|\left(\partial_{x}^{3} \partial \mathbf{v}, \partial_{x}^{2} \partial \varrho\right)\right|_{L^{2}}^{2} d s \\
\leq & C \int_{0}^{t} \mid\left(\partial_{x} \mathbf{v}_{t}, \partial_{x}^{2} \mathbf{v}_{t}, \partial\left(\frac{D \varrho}{d t}\right), \partial \partial_{x}\left(\frac{D \varrho}{d t}\right),\left.\partial \partial_{x}^{2}\left(\frac{D \varrho}{D t}\right)\right|_{L^{2}} ^{2} d s\right. \\
& +C \delta N(0, t)^{2} \\
& \leq C N(0,0)^{2}+C \delta N(0, t)^{2}+C \int_{0}^{t}\left|\partial_{x}^{2} \mathbf{v}_{t}, \partial \partial_{x}^{2}\left(\frac{D \varrho}{D t}\right)\right|_{L^{2}}^{2} d s
\end{aligned}
$$

which together with (53) yields

$$
\begin{gathered}
\left|\partial \partial_{x}^{2} \varrho\right|_{L^{2}}^{2}+\int_{0}^{t}\left|\left(\partial \partial_{x}^{2} \varrho, \partial \partial_{x}^{2}\left(\frac{D \varrho}{D t}\right), \partial \partial_{x}^{3} \mathbf{v}\right)\right|_{L^{2}}^{2} d s \\
\leq C N(0,0)^{2}+C \delta N(0, t)^{2}+C \int_{0}^{t}\left|\partial_{x}^{2} \mathbf{v}_{t}\right|_{L^{2}}^{2} d s
\end{gathered}
$$

By Lemma 3.6, $k=0, l=2$, and by (54), we have

$$
\begin{aligned}
& \left|\partial_{x}^{3} \varrho\right|_{L^{2}}^{2}+\int_{0}^{t}\left|\left(\partial_{x}^{3} \varrho, \partial_{x}^{3}\left(\frac{D \varrho}{D t}\right), \partial \partial_{x}^{3} \mathbf{v}\right)\right|_{L^{2}}^{2} d s \\
& \leq C N(0,0)^{2}+C \delta N(0, t)^{2}+C \int_{0}^{t}\left|\partial_{x}^{2} \mathbf{v}_{t}\right|_{L^{2}}^{2} d s
\end{aligned}
$$

Thus by Lemma $3.7, k=0, l=2$, we have

$$
\begin{aligned}
\int_{0}^{t}\left|\left(\partial_{x}^{4} \mathbf{v}, \partial_{x}^{3} \varrho\right)\right|_{L^{2}}^{2} d s & \leq C N(0,0)^{2}+C \delta N(0, t)^{2} \\
& +C \int_{0}^{t} \mid\left(\partial_{x}^{2} \mathbf{v}_{t},\left.\partial_{x}^{3}\left(\frac{D \varrho}{D t}\right)\right|_{L^{2}} ^{2} d s,\right.
\end{aligned}
$$

together it with (55), then we have got

$$
\begin{aligned}
\left|\partial_{x}^{3} \varrho\right|_{L^{2}}^{2} & +\int_{0}^{t}\left|\left(\partial_{x}^{3} \varrho, \partial_{x}^{3}\left(\frac{D \varrho}{D t}\right), \partial_{x}^{4} \mathbf{v}\right)\right|_{L^{2}}^{2} d s \leq C N(0,0)^{2} \\
& +C \delta N(0, t)^{2}+C \int_{0}^{t}\left|\partial_{x}^{2} \mathbf{v}_{t}\right|_{L^{2}}^{2} d s
\end{aligned}
$$

ii) By Lemma 3.1, $k=3$, we have

$$
\begin{aligned}
\left|\partial_{x}^{3}(\mathbf{v}, \mathbf{H})\right|_{L^{2}}^{2} & \leq C\left(\left|\left(\partial_{x} \varrho, \mathbf{v}_{t}, \mathbf{H}_{t}\right)\right|_{1}^{2}+\left|\left(S_{2}, S_{3}\right)\right|_{1}^{2}+\left|\left(\mathbf{v}, \partial_{x} \mathbf{H}\right)\right|_{L^{2}}^{2}\right) \\
& \leq C \delta N(0, t)^{2}+C\left|\varrho, \partial_{x}^{3} \varrho, \mathbf{v}, \mathbf{v}_{t}, \partial_{x} \mathbf{v}_{t}, \partial_{x} \mathbf{H}, \mathbf{H}_{t}, \partial_{x} \mathbf{H}_{t}\right|_{L^{2}}^{2},
\end{aligned}
$$

which together with (30)-(33), (46) and (56) implies

$$
\left|\partial_{x}^{3}(\mathbf{v}, \mathbf{H})\right|_{L^{2}}^{2} \leq C N(0,0)^{2}+C \delta N(0, t)^{2}+C \int_{0}^{t}\left|\partial_{x}^{2} \mathbf{v}_{t}\right|_{L^{2}}^{2} d s
$$


iii) By Lemma 3.1, $k=4$, we have

$$
\begin{gathered}
\int_{0}^{t}\left|\partial_{x}^{4} \mathbf{H}\right|_{L^{2}}^{2} d s \leq C \int_{0}^{t}\left|\mathbf{H}_{t}\right|_{2}^{2}+\left|S_{3}\right|_{2}^{2}+\left|\partial_{x} \mathbf{H}\right|_{L^{2}}^{2} d s \\
\leq C N(0,0)^{2}+C \delta N(0, t)^{2}+\int_{0}^{t}\left|\partial_{x}^{2} \mathbf{H}_{t}\right|_{L^{2}}^{2} d s,
\end{gathered}
$$

this together with (56) and (58), then it implies

$$
\begin{aligned}
& \left|\partial_{x}^{3}(\varrho, \mathbf{v}, \mathbf{H})\right|_{L^{2}}^{2}+\int_{0}^{t}\left|\left(\partial_{x}^{3} \varrho, \partial_{x}^{4} \mathbf{v}, \partial_{x}^{4} \mathbf{H}\right)(s)\right|_{L^{2}}^{2} d s \\
& \quad \leq C N(0,0)^{2}+C \delta N(0, t)^{2}+C \int_{0}^{t}\left|\partial_{x}^{2}\left(\mathbf{v}_{t}, \mathbf{H}_{t}\right)\right|_{L^{2}}^{2} d s .
\end{aligned}
$$

We can derive from Lemma 3.1 that $\left|\partial_{x}^{2}\left(\mathbf{v}_{t}, \mathbf{H}_{t}\right)\right|_{L^{2}}^{2} \leq C\left(\left|\left(\partial_{x} \varrho_{t}, \mathbf{v}_{t}, \mathbf{v}_{t t}, \partial_{x} \mathbf{H}_{t}, \mathbf{H}_{t t}, \partial_{t} S_{2}, \partial_{t} S_{3}\right)\right|_{L^{2}}^{2}\right.$.

Here with the help of $(19)_{1}$, we have

$$
\begin{aligned}
\int_{0}^{t}\left|\partial_{x} \varrho_{t}\right|_{L^{2}}^{2} d s & \leq C \int_{0}^{t}\left|\partial_{x}^{2} \varrho\right|_{L^{2}}^{2}+\left|\partial_{x}^{2} \mathbf{v}\right|_{L^{2}}^{2} d s \\
& \leq C(\varepsilon) \int_{0}^{t}\left|\partial_{x} \varrho, \partial_{x} \mathbf{v}\right|_{L^{2}}^{2} d s+\varepsilon \int_{0}^{t}\left|\partial_{x}^{3} \varrho, \partial_{x}^{4} \mathbf{v}\right|_{L^{2}}^{2} d s
\end{aligned}
$$

where the terms in the right-hand side can be absorbed by (46) and (59). Thus by (30)-(33), we obtain

$$
\int_{0}^{t}\left|\partial_{x}^{2}\left(\mathbf{v}_{t}, \mathbf{H}_{t}\right)\right|_{L^{2}}^{2} d s \leq C N(0,0)^{2}+C \delta N(0, t)^{2},
$$

which together with (59) implies (50).

\section{Part 3: Conclusion}

Combining the inequalities in Proposition 3.2 and Proposition 3.3, it yields

$$
\begin{aligned}
& \left|\left(\varrho, \partial_{x}^{3} \varrho, \mathbf{v}, \partial_{x}^{3} \mathbf{v}, \mathbf{H}, \partial_{x}^{3} \mathbf{H}\right)\right|_{L^{2}}^{2}+\int_{0}^{t}\left|\left(\partial_{x} \varrho, \partial_{x}^{3} \varrho, \partial_{x} \mathbf{v}, \partial_{x}^{4} \mathbf{v}, \partial_{x} \mathbf{H}, \partial_{x}^{4} \mathbf{H}\right)\right|_{L^{2}}^{2} d s \\
& \quad \leq C N(0,0)^{2}+C \delta N(0, t)^{2} .
\end{aligned}
$$

Thanks to (15), we can obtain that the left-hand side of (60) is equivalent to $N(0, t)^{2}$. Thus by the smallness of $\delta$, it finished the proof of Theorem 4 .

\section{Proof of Theorem 2}

In this section we shall prove the decay rates of the solution obtained in Theorem 1 to finish the proof of Theorem 2.

\section{Some elementary decay-in-time estimates}

We shall consider the convergence rates of the solution $(\varrho, \mathbf{v}, \mathbf{H})$ for the linearized problem (19). To use the $L^{p}-L^{q}$ estimates of the linear problem for the nonlinear problem
$(19)_{1}-(19)_{2}$, we rewrite the solution of $(19)_{1}-(19)_{2}$ as

$$
U(t)=E(t) U_{0}+\int_{0}^{t} E(t-s) F(U(s), \mathbf{H}(s)) d s,
$$

where we have used the notations

$$
U=[\varrho, \mathbf{v}]^{T}, U_{0}=\left[\varrho_{0}, \mathbf{v}_{0}\right]^{T}, F=\left[S_{1}, S_{2}\right]^{T},
$$

and the fact that $E(t)$ is the solution semigroup defined by $E(t)=e^{-t A}, t \geq 0$, with $A$ being a matrix-valued differential operator given by

$$
A=\left(\begin{array}{cc}
0 & \gamma \nabla^{T} \\
\gamma \nabla & -\mu \Delta-\mu \nabla \operatorname{div}
\end{array}\right) .
$$

The semigroup $E(t)$ has the following decay-in-time properties which can be found in (Kagei and Kobayashi 2005; Kobayashi 2002).

Lemma 3.8. There exist positive constants $C$ and $C_{0}$ such that for any $t \geq 1$ and $l=1,2$, we have i)

$$
\left|E(t) U_{0}\right|_{L^{2}} \leq C\left\{t^{-\frac{3}{4}}\left|U_{0}\right|_{L^{1}}+e^{-C_{0} t}\left(\left|\varrho_{0}\right|_{L^{2}}+\left|\mathbf{v}_{0}\right|_{L^{2}}\right)\right\},
$$

$\left|\partial_{\chi}^{l} E(t) U_{0}\right|_{L^{2}} \leq C\left\{t^{-\frac{3}{4}-\frac{l}{2}}\left|U_{0}\right|_{L^{1}}+e^{-C_{0} t}\left(\left|\varrho_{0}\right|_{l}+\left|\mathbf{v}_{0}\right|_{l-1}\right)\right\}$,

$$
\left|\partial_{x}^{3} E(t) U_{0}\right|_{L^{2}} \leq C\left\{t^{-\frac{15}{8}}\left|U_{0}\right|_{L^{1}}+e^{-C_{0} t}\left(\left|\varrho_{0}\right|_{3}+\left|\mathbf{v}_{0}\right|_{2}\right)\right\},
$$

and

$$
\left|E(t) U_{0}\right|_{L^{\infty}} \leq C\left\{t^{-\frac{3}{2}}\left|U_{0}\right|_{L^{1}}+e^{-C_{0} t}\left(\left|\varrho_{0}\right|_{2}+\left|\mathbf{v}_{0}\right|_{1}\right)\right\} .
$$

ii)

$\left|\partial_{x}^{l} \partial_{t} E(t) U_{0}\right|_{L^{2}} \leq C\left\{t^{-\frac{5}{4}-\frac{l}{2}}\left|U_{0}\right|_{L^{1}}+e^{-C_{0} t}\left(\left|\varrho_{0}\right|_{l}+\left|\mathbf{v}_{0}\right|_{l-1}\right)\right\}$.

Lemma 3.9. For any $k \in \mathbb{Z}, k \geq 0$, the following inequalities hold uniformly in $0<t \leq 1$,

$$
\begin{aligned}
& \left|E(t) U_{0}\right|_{H^{1} \cap L^{2}} \leq C\left|U_{0}\right|_{H^{1} \cap L^{2}}, \\
& \left|\partial_{X^{\prime}}^{k} \partial_{x} E(t) U_{0}\right|_{L^{2}} \leq C t^{-\frac{1}{2}}\left|U_{0}\right|_{H^{k+1} \cap H^{k}},
\end{aligned}
$$

and

$$
\left|E(t) U_{0}\right|_{L^{\infty}} \leq C t^{-(1-\bar{\varepsilon})}\left|U_{0}\right|_{H^{2} \cap H^{1}},
$$

for some $\bar{\varepsilon}>0$. Here $|\cdot|_{X \cap Y}=|\cdot|_{X}+|\cdot|_{Y}$. 
To treat the magnetic field, we notice that the solution to the heat equation $(19)_{3}$ has the following convergence estimates which one can refer to (Kagei and Kobayashi 2005; Kobayashi 2002).

Lemma 3.10. For the solution $\mathbf{H}$ to the heat equation (19) 3 with the initial data $\mathbf{H}(x, 0)=\mathbf{H}_{0}$ and the boundary condition $\left.\mathbf{H}\right|_{\left\{x_{3}=0\right\}}=0$, there exists a constant $C$ such that

$$
\begin{aligned}
|\mathbf{H}|_{L^{q}} \leq & C(1+t)^{-\frac{3}{2}\left(1-\frac{1}{q}\right)}\left|\mathbf{H}_{0}\right|_{L^{1} \cap L^{q}} \\
& +C \int_{0}^{t}(1+t-s)^{-\frac{3}{2}\left(1-\frac{1}{q}\right)}\left|S_{3}(\cdot, s)\right|_{L^{1} \cap L^{q}} d s
\end{aligned}
$$

and

$$
\begin{aligned}
\left|\partial_{x} \mathbf{H}\right|_{L^{2}} \leq & C(1+t)^{-\frac{5}{4}}\left|\mathbf{H}_{0}\right|_{L^{1} \cap H^{1}}+C \int_{t-1}^{t}(t-s)^{-\frac{1}{2}}\left|S_{3}\right|_{L^{2}} d s \\
& +C \int_{0}^{t-1}(1+t-s)^{-\frac{5}{4}}\left|S_{3}(\cdot, s)\right|_{L^{1}} d s,
\end{aligned}
$$

for any $t \geq 1,2 \leq q \leq+\infty$.

\section{Convergence rates of the lower-order derivatives}

Proposition 3.4. Let $s \geq 3$. Under the assumptions of Proposition 3.1, if there exists $\delta_{1}^{\prime}>0$ such that the initial data $\left(\varrho_{0}, \mathbf{v}_{0}, \mathbf{H}_{0}\right) \in H^{s} \cap L^{1}$ and

$$
\left|\left(\varrho_{0}, \mathbf{v}_{0}, \mathbf{H}_{0}\right)\right|_{H^{s} \cap L^{1}} \leq \delta_{1}^{\prime},
$$

then the solution $(\varrho, \mathbf{v}, \mathbf{H})$ of (19) satisfies

$$
|(\varrho, \mathbf{v}, \mathbf{H})(t)|_{L^{p}}=O\left(t^{-\frac{3}{2}\left(1-\frac{1}{p}\right)}\right), \forall p \in[2, \infty] .
$$

and

$$
\left|\partial_{x}(\varrho, \mathbf{v}, \mathbf{H})(t)\right|_{L^{2}}=O\left(t^{-\frac{5}{4}}\right)
$$

as $t \rightarrow \infty$.

Proof. We firstly estimate the $L^{2}$-estimate of the solution and its derivatives in Proposition 3.4. For simplicity, we shall introduce some notation in the sequel. Set

$$
M_{\sigma}^{s}(t)=\sup _{0 \leq \tau \leq t}(1+\tau)^{\sigma}\left|\partial_{x}^{s}(\varrho, \mathbf{v}, \mathbf{H})(\tau)\right|_{L^{2}},
$$

We will show that

$$
\begin{aligned}
M_{\frac{3}{4}}^{0}(t) & +M_{\frac{5}{4}}^{1}(t) \leq C\left\{\left|\left(\varrho_{0}, \mathbf{v}_{0}, \mathbf{H}_{0}\right)\right|_{L^{1}}+N(0, t)+M_{\frac{3}{4}}^{0}(t)^{2}\right. \\
& \left.+M_{\frac{5}{4}}^{1}(t)^{2}\right\}
\end{aligned}
$$

where $t \geq 0$. Thus we can derive the $L^{2}$ estimates in Proposition 3.4 from (73) by using a standard method under assumption of the smallness of initial data.

Now we shall consider the $L^{2}$ estimates of $U(t)$ and $\mathbf{H}(t)$ respectively. Thanks to Proposition 3.1, we only have to show the decay rate part of Proposition 3.4 for the case $t \geq 1$. Thus we assume that $t \geq 1$ and decompose $U(t)$ as

$$
\begin{aligned}
U(t)=E(t) U_{0} & +\int_{t-1}^{t} E(t-s) F(U(s), H(s)) d s \\
& +\int_{0}^{t-1} E(t-s) F(U(s), H(s)) d s \\
= & I_{0}(t)+I_{1}(t)+I_{2}(t) .
\end{aligned}
$$

By Lemma 3.8, we have

$$
\begin{aligned}
\left|\partial_{x}^{l} I_{0}(t)\right|_{L^{2}} \leq & C t^{-\frac{3}{4}-\frac{l}{2}}\left|U_{0}\right|_{L^{1} \cap H^{1}} \leq C(1+t)^{-\frac{3}{4}-\frac{l}{2}} \\
& \times\left\{\left|U_{0}\right|_{L^{1}}+N(0, t)\right\}, l=0,1 .
\end{aligned}
$$

Lemma 3.11. Under the assumptions of Proposition 3.4, the following inequalities hold for all $t \geq 0$,

$\left|S_{1}, \partial_{x} S_{1}, S_{2}, S_{3}\right|_{L^{2}} \leq C(1+t)^{-\frac{5}{4}}\left\{M_{\frac{5}{4}}^{1}(t)^{2}+N(0, t)^{2}\right\}$,

and

$\left|S_{1}, S_{2}, S_{3}\right|_{L^{1}} \leq C(1+t)^{-\frac{11}{8}}\left\{M_{\frac{3}{4}}^{0}(t)^{2}+M_{\frac{5}{4}}^{1}(t)^{2}+N(0, t)^{2}\right\}$.

Proof. Since by (16) and (17), we have

$$
\begin{aligned}
\left|S_{1}, S_{3}\right|_{L^{2}} & \leq C|(\varrho, \mathbf{v}, \mathbf{H})|_{L^{\infty}}\left|\partial_{x}(\varrho, \mathbf{v}, \mathbf{H})\right|_{L^{2}} \\
& \leq C(1+t)^{-\frac{5}{4}} M_{\frac{5}{4}}^{1}(t) N(0, t) \\
& \leq C(1+t)^{-\frac{5}{4}}\left\{M_{\frac{5}{4}}^{1}(t)^{2}+N(0, t)^{2}\right\},
\end{aligned}
$$

$$
\begin{aligned}
\left|\partial_{x} S_{1}\right|_{L^{2}} & \leq C\left|\partial_{x}(\varrho, \mathbf{v})\right|_{L^{\infty}}\left|\partial_{x}(\varrho, \mathbf{v})\right|_{L^{2}}+|\varrho, \mathbf{v}|_{L^{6}}\left|\partial_{x}^{2}(\varrho, \mathbf{v})\right|_{L^{6}}^{\frac{1}{2}}\left|\partial_{x}^{2}(\varrho, \mathbf{v})\right|_{L^{2}}^{\frac{1}{2}} \\
& \leq C(1+t)^{-\frac{5}{4}} M_{\frac{5}{4}}^{1}(t) N(0, t)+\left|\partial_{x}(\varrho, \mathbf{v})\right|_{L^{2}}\left|\partial_{x}^{2} \varrho, \partial_{x}^{3} \varrho, \partial_{x}^{2} \mathbf{v}, \partial_{x}^{3} \mathbf{v}\right|_{L^{2}} \\
& \leq C(1+t)^{-\frac{5}{4}}\left\{M_{\frac{5}{4}}^{1}(t)^{2}+N(0, t)^{2}\right\},
\end{aligned}
$$

and

$$
\begin{aligned}
\left|S_{2}\right|_{L^{2}} & \leq C|(\varrho, \mathbf{v}, \mathbf{H})|_{L^{\infty}}\left|\partial_{x}(\varrho, \mathbf{v}, \mathbf{H})\right|_{L^{2}}+C\left|\partial_{x}^{2} \mathbf{v}\right|_{L^{2}}^{\frac{1}{2}}\left|\partial_{x}^{2} \mathbf{v}\right|_{L^{6}}^{\frac{1}{2}}|\varrho|_{L^{6}} \\
& \leq C(1+t)^{-\frac{5}{4}}\left\{M_{\frac{5}{4}}^{1}(t)^{2}+N(0, t)^{2}\right\},
\end{aligned}
$$


together with these inequalities, we can easily get (76). Moreover, (77) follows from

$$
\begin{aligned}
\left|S_{1}, S_{3}\right|_{L^{1}} \leq & C|(\varrho, \mathbf{v}, \mathbf{H})|_{L^{2}}\left|\partial_{x}(\varrho, \mathbf{v}, \mathbf{H})\right|_{L^{2}} \\
\leq & C(1+t)^{-2} M_{\frac{3}{4}}^{0}(t) M_{\frac{5}{4}}^{1}(t) \leq C(1+t)^{-2} \\
& \times\left\{M_{\frac{3}{4}}^{0}(t)^{2}+M_{\frac{5}{4}}^{1}(t)^{2}\right\},
\end{aligned}
$$

and

$$
\begin{aligned}
\left|S_{2}\right|_{L^{1}} \leq & C|(\varrho, \mathbf{v}, \mathbf{H})|_{L^{2}}\left|\left(\partial_{x} \varrho, \partial_{x} \mathbf{v}, \partial_{x} \mathbf{H}\right)\right|_{L^{2}}+\left.C|\varrho|_{L^{2}}|| \partial_{x}^{2} \mathbf{v}\right|_{L^{2}} \\
\leq & C(1+t)^{-2} M_{\frac{3}{4}}^{0}(t) M_{\frac{5}{4}}^{1}(t)+\left.C|\varrho|_{L^{2}}|| \partial_{x} \mathbf{v}\right|_{L^{2}} ^{\frac{1}{2}}\left|\partial_{x}^{3} \mathbf{v}\right|_{L^{2}}^{\frac{1}{2}} \\
\leq & C(1+t)^{-2}\left\{M_{\frac{3}{4}}^{0}(t)^{2}+M_{\frac{5}{4}}^{1}(t)^{2}\right\} \\
& +C(1+t)^{-\frac{11}{8}} M_{\frac{3}{4}}^{0}(t) M_{\frac{5}{4}}^{1}(t)^{\frac{1}{2}} N^{\frac{1}{2}}(0, t) \\
\leq & C(1+t)^{-\frac{11}{8}}\left\{M_{\frac{3}{4}}^{0}(t)^{2}+M_{\frac{5}{4}}^{1}(t)^{2}+N(0, t)^{2}\right\} .
\end{aligned}
$$

Now we have to estimate $I_{1}(t)$ and $I_{2}(t)$. For $l=0,1$, and by Lemma 3.9 and Lemma 3.11, we have

$$
\begin{aligned}
\left|\partial_{x}^{l} I_{1}(t)\right|_{L^{2}} & \leq C \int_{t-1}^{t}(t-\tau)^{-\frac{l}{2}}\left(\left|S_{1}\right|_{1}+\left|S_{2}\right|_{L^{2}}\right) d \tau \\
& \leq C \int_{t-1}^{t}(t-\tau)^{-\frac{l}{2}}(1+\tau)^{-\frac{5}{4}} d \tau\left\{M_{\frac{5}{4}}^{1}(t)^{2}+N(0, t)^{2}\right\} \\
& \leq C(1+t)^{-\frac{5}{4}}\left\{M_{\frac{5}{4}}^{1}(t)^{2}+N(0, t)^{2}\right\},
\end{aligned}
$$

where the last inequality is obtained by taking $l=1$ and $l=2$ respectively.

For $I_{2}(t)$, we can derive from Lemma 3.8 and Lemma 3.9 that

$$
\begin{aligned}
\left|\partial_{x}^{l} I_{2}(t)\right|_{L^{2}} \leq & C \int_{0}^{t-1}(t-\tau)^{-\frac{3}{2}-\frac{l}{2}}|F(U(\tau), \mathbf{H}(\tau))|_{L^{1}} \\
& +e^{-C_{0}(t-\tau)}|U(\tau)|_{1} d \tau \\
\leq & C \int_{0}^{t-1}(t-\tau)^{-\frac{3}{2}-\frac{l}{2}}(1+\tau)^{-\frac{11}{8}} d \tau \\
& \times\left\{M_{\frac{3}{4}}^{0}(t)^{2}+M_{\frac{5}{4}}^{1}(t)^{2}+N(0, t)^{2}\right\} \\
& +C \int_{0}^{t-1} e^{-C_{0}(t-\tau)}(1+\tau)^{-\frac{5}{4}} d \tau \\
& \times\left\{M_{\frac{5}{4}}^{1}(t)^{2}+N(0, t)^{2}\right\} \\
\leq & C(1+t)^{-\frac{3}{4}-\frac{l}{2}}\left\{M_{\frac{3}{4}}^{0}(t)^{2}+M_{\frac{5}{4}}^{1}(t)^{2}+N(0, t)^{2}\right\} .
\end{aligned}
$$

Now we turn to do the estimates for $\mathbf{H}(t)$. By (71) in Lemma 3.10, we can deduce that

$$
\begin{aligned}
|\mathbf{H}|_{L^{2}} \leq & C(1+t)^{-\frac{3}{4}}\left|\mathbf{H}_{0}\right|_{L^{1} \cap L^{2}}+\int_{0}^{t}(1+t-s)^{-\frac{3}{4}}\left|S_{3}\right|_{L^{1} \cap L^{2}} d s \\
\leq & C(1+t)^{-\frac{3}{4}}\left\{\left|\mathbf{H}_{0}\right|_{L^{1}}+N(0, t)\right\} \\
& +C\left\{M_{\frac{3}{4}}^{0}(t)^{2}+M_{\frac{5}{4}}^{1}(t)^{2}+N(0, t)^{2}\right\} \\
& \times \int_{0}^{t}(1+t-s)^{-\frac{3}{4}}(1+s)^{-\frac{11}{8}} d s \\
\leq & C(1+t)^{-\frac{3}{4}}\left\{\left|\mathbf{H}_{0}\right|_{L^{1}}+N(0, t)+M_{\frac{3}{4}}^{0}(t)^{2}+M_{\frac{5}{4}}^{1}(t)^{2}\right\},
\end{aligned}
$$

and similarly by (72), we have

$$
\begin{aligned}
\left|\partial_{x} \mathbf{H}\right|_{L^{2}} \leq & C(1+t)^{-\frac{5}{4}}\left|\mathbf{H}_{0}\right|_{L^{1} \cap H^{1}}+\int_{t-1}^{t}(t-s)^{-\frac{1}{2}}\left|S_{3}\right|_{L^{2}} d s \\
& +\int_{0}^{t-1}(1+t-s)^{-\frac{5}{4}}\left|S_{3}\right|_{L^{1}} d s \\
\leq & C(1+t)^{-\frac{5}{4}}\left\{\left|\mathbf{H}_{0}\right|_{L^{1}}+N(0, t)+M_{\frac{3}{4}}^{0}(t)^{2}+M_{\frac{5}{4}}^{1}(t)^{2}\right\} .
\end{aligned}
$$

Combining these with (75), (78) and (79), it implies (73). Now we shall do the $L^{\infty}$ estimate. Define

$$
M_{\infty}(t)=\sup _{0 \leq \tau \leq t}(1+\tau)^{\frac{3}{2}}|(\varrho, \mathbf{v}, \mathbf{H})(\tau)|_{L^{\infty}}
$$

and

$\mathcal{M}_{\sigma}^{s}(t)=\sum_{k=0}^{s} M_{\sigma}^{k}(t)=\sum_{k=0}^{s} \sup _{0 \leq \tau \leq t}(1+\tau)^{\sigma}\left|\partial_{x}^{k}(\varrho, \mathbf{v}, \mathbf{H})(\tau)\right|_{L^{2}}$.

By Lemma 3.8, we have

$$
\begin{aligned}
\left|I_{0}\right|_{L^{\infty}} & \leq C t^{-\frac{3}{2}}\left(\left|U_{0}\right|_{L^{1}}+\left|\varrho_{0}\right|_{2}+\left|\mathbf{v}_{0}\right|_{1}\right) \\
& \leq C(1+t)^{-\frac{3}{2}}\left(\left|U_{0}\right|_{L^{1}}+N(0, t)\right) .
\end{aligned}
$$

To estimate $I_{1}(t)$ and $I_{2}(t)$, we state the following estimates for $S_{1}$ and $S_{2}$.

Lemma 3.12. Under the assumptions of Proposition 3.4, there exists a sufficiently small constant $\widetilde{\varepsilon}>0$ such that for any $0<\varepsilon<\widetilde{\varepsilon}$, the following inequalities hold uniformly in $t \geq 0$.

i)

$\left|S_{1}\right|_{2},\left|S_{2}\right|_{1} \leq C(1+t)^{-\frac{3}{2}}\left\{M_{\infty}(t)^{2}+M_{\frac{5}{4}}^{1}(t)^{2}+\mathcal{M}_{\frac{3}{4}-\varepsilon}^{3}(t)^{2}\right\}$, 
and

$$
\left|S_{1}, S_{2}\right|_{L^{1}} \leq C t^{-\left(\frac{7}{4}-\varepsilon\right)}\left\{M_{\frac{3}{4}}^{0}(t)^{2}+M_{\frac{5}{4}}^{1}(t)^{2}+\mathcal{M}_{\frac{3}{4}-\varepsilon}^{3}(t)^{2}\right\}
$$

ii)

$$
\left|S_{1}\right|_{3},\left|S_{2}\right|_{2} \leq C(1+t)^{-\frac{3}{2}}\left\{M_{\infty}(t)^{2}+M_{\frac{5}{4}}^{1}(t)^{2}+\mathcal{K}_{\frac{3}{4}-\varepsilon}^{4}(t)^{2}\right\} \text {, }
$$

and

$$
\left|\partial_{t} S_{1}\right|_{1},\left|\partial_{t} S_{2}\right|_{L_{2}} \leq C(1+t)^{-\frac{5}{4}}\left\{\mathcal{K}_{\frac{3}{4}-\varepsilon}^{4}(t)^{2}+M_{\infty}(t)^{2}+M_{\frac{5}{4}}^{1}(t)^{2}\right\},
$$

here we define

$$
\begin{aligned}
\mathcal{K}_{\sigma}^{s}(t)= & \mathcal{M}_{\sigma}^{s}(t)+\sum_{j=1}^{\left[\frac{s}{2}\right]} \sup _{0 \leq \tau \leq t}(1+\tau)^{\sigma}\left|\partial_{\tau}^{j}(\mathbf{v}, \mathbf{H})(\tau)\right|_{s-2 j} \\
& +\sum_{j=1}^{\left[\frac{s+1}{2}\right]} \sup _{0 \leq \tau \leq t}(1+\tau)^{\sigma}\left|\partial_{\tau}^{j} \varrho(\tau)\right|_{s+1-2 j} .
\end{aligned}
$$

Proof. For $l=0,1,2$, we have

$$
\begin{aligned}
\left|\partial_{x}^{l} S_{1}\right|_{L^{2}} \leq & C\left|\partial_{x}^{l+1}(\varrho \mathbf{v})\right|_{L^{2}} \\
\leq & C\left(|\varrho, \mathbf{v}|_{L^{\infty}}\left|\partial_{x}(\varrho, \mathbf{v})\right|_{2}+\left|\partial_{x}(\varrho, \mathbf{v})\right|_{L^{2}}^{\frac{1}{2}}\left|\partial_{x}^{2}(\varrho, \mathbf{v})\right|_{1}^{\frac{3}{2}}\right) \\
\leq & C\left\{(1+t)^{-\left(\frac{9}{4}-\varepsilon\right)} M_{\infty}(t) \mathcal{M}_{\frac{3}{4}-\varepsilon}^{3}(t)\right. \\
& \left.+(1+t)^{-\left(\frac{7}{4}-\frac{3}{2} \varepsilon\right)} M_{\frac{5}{4}}^{1}(t)^{\frac{1}{2}} \mathcal{M}_{\frac{3}{4}-\varepsilon}^{3}(t)^{\frac{3}{2}}\right\} \\
\leq & C(1+t)^{-\frac{3}{2}}\left\{M_{\infty}(t)^{2}+M_{\frac{5}{4}}^{1}(t)^{2}+\mathcal{M}_{\frac{3}{4}-\varepsilon}^{3}(t)^{2}\right\}
\end{aligned}
$$

and for $l=0,1$, we have

$$
\begin{aligned}
\left|\partial_{x}^{l} S_{2}\right|_{L^{2}} \leq & C\left(|\varrho, \mathbf{v}, \mathbf{H}|_{L^{\infty}}\left|\partial_{x}(\varrho, \mathbf{v}, \mathbf{H})\right|_{2}\right. \\
& \left.+\left|\partial_{x}(\varrho, \mathbf{v}, \mathbf{H})\right|_{L^{2}}^{\frac{1}{2}}\left|\partial_{x}(\varrho, \mathbf{v}, \mathbf{H})\right|_{1}^{\frac{3}{2}}\right) \\
\leq & C(1+t)^{-\frac{3}{2}}\left\{M_{\infty}^{2}+M_{\frac{5}{4}}^{1}(t)^{2}+\mathcal{M}_{\frac{3}{4}-\varepsilon}^{3}(t)^{2}\right\}
\end{aligned}
$$

Then we get (81). Similarly,

$\left|S_{1}\right|_{L^{1}} \leq C|\varrho, \mathbf{v}|_{L^{2}}\left|\partial_{x}(\varrho, \mathbf{v})\right|_{L^{2}} \leq C(1+t)^{-2}\left\{M_{\frac{3}{4}}^{0}(t)^{2}+M_{\frac{5}{4}}^{1}(t)^{2}\right\}$, and

$$
\begin{aligned}
\left|S_{2}\right|_{L^{1}} \leq & C\left(|\varrho, \mathbf{v}, \mathbf{H}|_{L^{2}}\left|\partial_{x}(\varrho, \mathbf{v}, \mathbf{H})\right|_{L^{2}}+|\varrho|_{L^{2}}\left|\partial_{x}^{2} \mathbf{v}\right|_{L^{2}}\right) \\
\leq & C\left(|\varrho, \mathbf{v}, \mathbf{H}|_{L^{2}}\left|\partial_{x}(\varrho, \mathbf{v}, \mathbf{H})\right|_{L^{2}}+|\varrho|_{L^{2}}\left|\partial_{x} \mathbf{v}\right|_{L^{2}}^{\frac{1}{2}}\left|\partial_{x}^{3} \mathbf{v}\right|_{L^{2}}^{\frac{1}{2}}\right) \\
\leq & C\left\{(1+t)^{-2} M_{\frac{3}{4}}^{0}(t) M_{\frac{5}{4}}^{1}(t)\right. \\
& \left.+(1+t)^{-\left(\frac{7}{4}-\varepsilon\right)} M_{\frac{3}{4}}^{0}(t) M_{\frac{5}{4}}^{1}(t) \mathcal{M}_{\frac{3}{4}-\varepsilon}^{3}(t)\right\} \\
\leq & C(1+t)^{-\left(\frac{7}{4}-\varepsilon\right)}\left\{M_{\frac{3}{4}}^{0}(t)^{2}+M_{\frac{5}{4}}^{1}(t)^{2}+\mathcal{M}_{\frac{3}{4}-\varepsilon}^{3}(t)^{2}\right\} .
\end{aligned}
$$

Thus we obtain (82). We shall get the inequalities in ii) in the similar way. Here we only estimate $\left|\partial_{x}^{3} S_{1}\right|_{L^{2}}$ and $\left|\partial_{t}(\varrho \Delta \mathbf{v})\right|_{L^{2}}$ as follows.

$$
\begin{aligned}
\left|\partial_{x}^{3} S_{1}\right|_{L^{2}} & \leq C\left(|\varrho, \mathbf{v}|_{L^{\infty}}\left|\partial_{x}^{4}(\varrho, \mathbf{v})\right|_{L^{2}}\right. \\
& \leq C\left\{(1+t)^{-\left(\frac{9}{4}-\varepsilon\right)} M_{\infty}(t) \mathcal{K}_{\frac{3}{4}-\varepsilon}^{4}(t)\right. \\
& \leq C(1+t)^{-\frac{3}{2}}\left\{M_{\infty}^{2}+\mathcal{K}_{\frac{3}{4}-\varepsilon}^{4}(t)^{2}\right\},
\end{aligned}
$$

and

$$
\begin{aligned}
& \left|\partial_{t}(\varrho \Delta \mathbf{v})\right|_{L^{2}} \leq|\varrho|_{L^{\infty}}\left|\Delta \mathbf{v}_{t}\right|_{L^{2}}+\left|\varrho_{t}\right|_{1}\left|\partial_{x}^{3} \mathbf{v}\right|_{L^{2}} \\
& \leq C\left\{(1+t)^{-\left(\frac{9}{4}-\varepsilon\right)} M_{\infty}(t) \mathcal{K}_{\frac{3}{4}-\varepsilon}^{4}(t)\right. \\
& +(1+t)^{-\left(\frac{3}{2}-2 \varepsilon\right)} \mathcal{K}_{\frac{3}{4}-\varepsilon}^{4}(t)^{2} \\
& \leq C(1+t)^{-\frac{5}{4}}\left\{M_{\infty}^{2}+\mathcal{K}_{\frac{3}{4}-\varepsilon}^{4}(t)^{2}\right\} .
\end{aligned}
$$

By Lemma 3.9 and (81), we have

$$
\begin{aligned}
\left|I_{1}\right|_{L^{\infty}} \leq & C \int_{t-1}^{t}(t-\tau)^{-(1-\bar{\varepsilon})}\left(\left|S_{1}\right|_{2}+\left|S_{2}\right|_{1}\right) d \tau \\
\leq & C\left\{M_{\infty}^{2}+M_{\frac{5}{4}}^{1}(t)^{2}+\mathcal{M}_{\frac{3}{4}-\varepsilon}^{3}(t)^{2}\right\} \\
& \times \int_{t-1}^{t}(t-\tau)^{-(1-\bar{\varepsilon})}(1+\tau)^{-\frac{3}{2}} d \tau \\
\leq & C(1+t)^{-\frac{3}{2}}\left\{M_{\infty}^{2}+M_{\frac{5}{4}}^{1}(t)^{2}+\mathcal{M}_{\frac{3}{4}-\varepsilon}^{3}(t)^{2}\right\} .
\end{aligned}
$$


As for $I_{2}$, we can deduce from Lemma 3.8 that

$$
\begin{aligned}
\left|I_{2}(t)\right|_{L^{\infty}} \leq C & \left\{\int_{0}^{t-1}(t-\tau)^{-\frac{3}{2}}\left|\left(S_{1}, S_{2}\right)(\tau)\right|_{L^{1}}\right. \\
& \left.+\int_{0}^{t-1} e^{-C_{0}(t-\tau)}\left(\left|S_{1}(\tau)\right|_{2}+\left|S_{2}(\tau)\right|_{1}\right) d \tau\right\} \\
\leq C & \left\{M_{\frac{3}{4}}^{0}(t)^{2}+M_{\frac{5}{4}}^{1}(t)^{2}+\mathcal{M}_{\frac{3}{4}-\varepsilon}^{3}(t)^{2}\right\} \\
& \times \int_{0}^{t-1}(t-\tau)^{-\frac{3}{2}}(1+\tau)^{-\left(\frac{7}{4}-\varepsilon_{1}\right)} d \tau \\
& +C\left\{M_{\infty}^{2}+M_{\frac{5}{4}}^{1}(t)^{2}+\mathcal{M}_{\frac{3}{4}-\varepsilon}^{3}(t)^{2}\right\} \\
& \times \int_{0}^{t-1} e^{-C_{0}(t-\tau)}(1+\tau)^{-\frac{3}{2}} d \tau \\
\leq C & +t)^{-\frac{3}{2}}\left\{M_{\infty}^{2}+M_{\frac{3}{4}}^{0}(t)^{2}+M_{\frac{5}{4}}^{1}(t)^{2}\right. \\
& \left.+\mathcal{M}_{\frac{3}{4}-\varepsilon}^{3}(t)^{2}\right\} .
\end{aligned}
$$

At last, we have to estimate $|\mathbf{H}|_{L^{\infty}}$ to finish the $L^{\infty}$ estimate. By Lemma 3.3, For $q=\infty$, we have

$$
\begin{aligned}
&|\mathbf{H}|_{L^{\infty}} \\
& \leq C(1+t)^{-\frac{3}{2}}\left|\mathbf{H}_{0}\right|_{L^{1} \cap L^{\infty}}+C \int_{0}^{t}(1+t-\tau)^{-\frac{3}{2}}\left|S_{3}(\tau)\right|_{L^{1} \cap L^{\infty}} d \tau \\
& \leq C(1+t)^{-\frac{3}{2}}\left\{\left|\mathbf{H}_{0}\right|_{L^{1}}+N(0, t)\right\} \\
&+C\left\{M_{\frac{3}{4}}^{0}(t)^{2}+M_{\frac{5}{4}}^{1}(t)^{2}+M_{\infty}(t)^{2}+N(0, t)^{2}\right\} \\
& \times \int_{0}^{t}(1+t-\tau)^{-\frac{3}{2}}(1+\tau)^{-\frac{3}{2}} d \tau \\
& \leq C(1+t)^{-\frac{3}{2}}\left\{\left|\mathbf{H}_{0}\right|_{L^{1}}+N(0, t)+M_{\infty}(t)^{2}+M_{\frac{3}{4}}^{0}(t)^{2}+M_{\frac{5}{4}}^{1}(t)^{2}\right\},
\end{aligned}
$$

where we have used

$$
\left|S_{3}\right|_{L^{1}} \leq C(1+t)^{-2}\left\{M_{\frac{3}{4}}^{0}(t)^{2}+M_{\frac{5}{4}}^{1}(t)^{2}\right\},
$$

which is in the proof of Lemma 3.12, and

$\left|S_{3}\right|_{L^{\infty}} \leq|\mathbf{v}, \mathbf{H}|_{L^{\infty}}|\mathbf{v}, \mathbf{H}|_{3} \leq C(1+t)^{-\frac{3}{2}}\left\{M_{\infty}(t)^{2}+N(0, t)^{2}\right\}$.

Thus, (80) and (85)-(87) imply

$$
\begin{aligned}
M_{\infty} \leq C\left\{\left|\varrho_{0}, \mathbf{v}_{0}, \mathbf{H}_{0}\right|_{L^{1}}\right. & +N(0, t)+M_{\infty}(t)^{2}+M_{\frac{3}{4}}^{0}(t)^{2} \\
& \left.+M_{\frac{5}{4}}^{1}(t)^{2}+\mathcal{M}_{\frac{3}{4}-\varepsilon}^{3}(t)^{2}\right\} .
\end{aligned}
$$

This together with (73), the estimate of $\mathcal{K}_{\frac{3}{4}-\varepsilon}^{4}(t)$ in Proposition 3.5 in the next subsection, it yields the $L^{\infty}$ estimate in Proposition 3.4. Hence by interpolation, we get the $L^{p}$-estimate for $2 \leq p \leq \infty$. The proof of Proposition 3.4 is complete.

\section{Convergence rates of the higher-order derivatives}

Now we shall do the estimates for the higher-order derivatives to finish the proof of Theorem 2 .

Proposition 3.5. There exists a positive number $\widetilde{\varepsilon}$ such that under the assumptions of Proposition 3.4, the solution $(\varrho, \mathbf{v}, \mathbf{H})$ of (19) satisfies

$$
\begin{aligned}
|(\varrho, \mathbf{v}, \mathbf{H})(t)|_{s} \leq & C\left(\left|\varrho_{0}, \mathbf{v}_{0}, \mathbf{H}_{0}\right|_{L^{1}}+\left|\varrho_{0}, \mathbf{v}_{0}, \mathbf{H}_{0}\right|_{s}\right) \\
& \times(1+t)^{-\left(\frac{3}{4}-\varepsilon_{1}\right)}, \forall 0<\varepsilon_{1}<\widetilde{\varepsilon}
\end{aligned}
$$

for all $t \geq 0$.

Set

$$
\begin{aligned}
\mathcal{L}_{\sigma}^{s}= & \left\{\int_{0}^{t}(1+\tau)^{2 \sigma}\left(\left|\partial_{x}(\mathbf{v}, \mathbf{H})\right|_{s+1}+\left|\partial_{x} \varrho\right|_{s}\right) d \tau\right. \\
& \left.+\sum_{j=1}^{\left[\frac{s+1}{2}\right]} \int_{0}^{t}(1+\tau)^{2 \sigma}\left|\partial_{\tau}^{j}(\varrho, \mathbf{v}, \mathbf{H})\right|_{s+1-2 j} d \tau\right\},
\end{aligned}
$$

and

$$
\begin{aligned}
& \mathcal{N}_{\sigma}^{s}=\left\{\sum_{j=0}^{\left[\frac{s-1}{2}\right]} \sup _{0 \leq \tau \leq t}(1+\tau)^{2 \sigma}\left|\partial_{\tau}^{j} S_{1}(\tau)\right|_{s-1-2 j}^{2}\right. \\
& +\sum_{j=0}^{\left[\frac{s}{2}\right]} \int_{0}^{t}(1+\tau)^{2 \sigma}\left|\partial_{\tau}^{j}(\varrho \operatorname{divv})(\tau)\right|_{s-2 j}^{2} d \tau \\
& +\sum_{j=0}^{\left[\frac{s-1}{2}\right]} \int_{0}^{t}(1+\tau)^{2 \sigma}\left|\partial_{\tau}^{j}(\mathbf{v} \cdot \nabla \varrho)\right|_{s-1-2 j}^{2} d \tau \\
& +\sum_{j=0}^{\left[\frac{s-2}{2}\right]} \sup _{0 \leq \tau \leq t}(1+\tau)^{2 \sigma}\left|\partial_{\tau}^{j}\left(S_{2}, S_{3}\right)(\tau)\right|_{s-2-2 j}^{2} \\
& +\sum_{j=0}^{\left[\frac{s-1}{2}\right]} \int_{0}^{t}(1+\tau)^{2 \sigma}\left|\partial_{\tau}\left(S_{2}, S_{3}\right)\right|_{s-1-2 j}^{2} d \tau \\
& +\sum_{0 \leq k+2 j \leq s} \int_{0}^{t}(1+\tau)^{2 \sigma}\left|\left\langle\partial^{k} \partial_{\tau}^{j} S, \partial^{k} \partial_{\tau}^{j} V\right\rangle\right| d \tau \\
& +\sum_{0 \leq k+2 j \leq s-1} \int_{0}^{t}(1+\tau)^{2 \sigma}\left|\left\langle\partial^{k} \partial_{\tau}^{j} S, \partial^{k} \partial_{\tau}^{j+1} V\right\rangle\right| d \tau \\
& +\sum_{0 \leq k+l+2 j \leq s-1} \int_{0}^{t}(1+\tau)^{2 \sigma} \gamma \mu \mid\left\langle\partial^{k} \partial_{3}^{l+1} \partial_{\tau}^{j}\right. \\
& \left.\left.\times(\mathbf{v} \cdot \nabla \varrho), \partial^{k} \partial_{3}^{l+1} \partial_{\tau}^{j} \varrho\right\rangle \mid d \tau\right\}^{\frac{1}{2}},
\end{aligned}
$$


where $S=\left(S_{1}, S_{2}, S_{3}\right)^{T}$ and $V=(\varrho, \mathbf{v}, \mathbf{H})^{T}$. As in the proof of (11.6) in (Kagei and Kobayashi 2005), we have the following inequality in the similar way:

Lemma 3.13. Under the assumptions of Proposition 3.4, the following inequality holds uniformly in $t \geq 0$ and for $\sigma \geq \frac{1}{2}$

$$
\begin{aligned}
\mathcal{K}_{\sigma}^{\mathcal{S}}(t)^{2}+\mathcal{L}_{\sigma}^{\mathcal{S}}(t)^{2} \leq & C\left|\varrho_{0}, \mathbf{v}_{0}, \mathbf{H}_{0}\right|_{s}^{2}+C \mathcal{L}_{0}^{s}(t)^{2} \\
& +C\left\{\mathcal{N}_{\sigma}^{s}(t)^{2}+\int_{0}^{t}(1+\tau)^{2 \sigma-1}|(\varrho, \mathbf{v}, \mathbf{H})(\tau)|_{L^{2}}^{2} d \tau\right\} .
\end{aligned}
$$

Proof. Here we only estimate the magnetic field. We shall show that,

$$
\begin{aligned}
(1+t)^{2 \sigma}|\mathbf{H}(t)|_{s} & +\sum_{j=1}^{\left[\frac{s}{2}\right]}(1+t)^{2 \sigma}\left|\partial_{t}^{j} \mathbf{H}(t)\right|_{s-2 j} \\
& +\sum_{j=1}^{\left[\frac{s+1}{2}\right]} \int_{0}^{t}(1+\tau)^{2 \sigma}\left|\partial_{\tau}^{j} \mathbf{H}(t)\right|_{s+1-2 j} d \tau \\
\leq & C\left\{\left|\varrho_{0}, \mathbf{v}_{0}, \mathbf{H}_{0}\right|_{s}^{2}+\mathcal{L}_{0}^{s}(t)^{2}+\mathcal{N}_{\sigma}^{s}(t)^{2}\right. \\
& \left.+\int_{0}^{t}(1+\tau)^{2 \sigma-1}|\mathbf{H}|_{L^{2}}^{2} d \tau\right\} .
\end{aligned}
$$

Then we estimate these as follows:

i) Taking $\partial_{t}^{j}$ to $(19)_{3}$, multiplying $(1+t)^{2 \sigma} \partial_{t}^{j} \mathbf{H}$, and integrating on $\mathbb{R}_{+}^{3}$, it implies

$$
\begin{aligned}
& \frac{1}{2} \frac{d}{d t} \int(1+t)^{2 \sigma}\left|\partial_{t}^{j} \mathbf{H}(t)\right|^{2} d x+(1+t)^{2 \sigma} \int\left|\partial_{x} \partial_{\tau}^{j} \mathbf{H}\right|^{2} d x \\
& \quad=\sigma(1+t)^{2 \sigma-1} \int\left|\partial_{t}^{j} \mathbf{H}(t)\right|^{2} d x+(1+t)^{2 \sigma}\left\langle\partial_{t}^{j} S_{3}, \partial_{t}^{j} \mathbf{H}\right\rangle .
\end{aligned}
$$

Integrating this from 0 to $t$ yields

$$
\begin{aligned}
& (1+t)^{2 \sigma}\left|\partial_{t}^{j} \mathbf{H}(t)\right|_{L^{2}}^{2}+\int_{0}^{t}(1+\tau)^{2 \sigma}\left|\partial_{x} \partial_{\tau}^{j} \mathbf{H}\right|_{L^{2}}^{2} d \tau \\
& \leq C\left|\varrho_{0}, \mathbf{v}_{0}, \mathbf{H}_{0}\right|_{s}^{2}+C \int_{0}^{t} \sigma(1+\tau)^{2 \sigma-1}\left|\partial_{\tau}^{j} \mathbf{H}\right|_{L^{2}}^{2} d \tau \\
& \quad+C \int_{0}^{t}(1+\tau)^{2 \sigma}\left|\left\langle\partial_{\tau}^{j} S_{3}, \partial_{\tau}^{j} \mathbf{H}\right\rangle\right| d \tau,
\end{aligned}
$$

ii) Taking $\partial_{t}^{j}$ to $(19)_{3}$, multiplying $(1+t)^{2 \sigma} \partial_{t}^{j+1} \mathbf{H}$, and integrating on $\mathbb{R}_{+}^{3}$, it holds

$$
\begin{aligned}
& \int(1+t)^{2 \sigma}\left|\partial_{t}^{j+1} \mathbf{H}(t)\right|^{2} d x+\frac{1}{2} \frac{d}{d t} \int(1+t)^{2 \sigma}\left|\partial_{x} \partial_{t}^{j} \mathbf{H}(t)\right|^{2} d x \\
& =\sigma(1+t)^{2 \sigma-1} \int\left|\partial_{x} \partial_{t}^{j} \mathbf{H}(t)\right|^{2} d x+(1+t)^{2 \sigma}\left\langle\partial_{t}^{j} S_{3}, \partial_{t}^{j+1} \mathbf{H}\right\rangle .
\end{aligned}
$$

By integrating this from 0 to $t$, we get

$$
\begin{aligned}
& (1+t)^{2 \sigma}\left|\partial_{x} \partial_{t}^{j} \mathbf{H}(t)\right|_{L^{2}}^{2}+\int_{0}^{t}(1+\tau)^{2 \sigma}\left|\partial_{\tau}^{j+1} \mathbf{H}\right|_{L^{2}}^{2} d \tau \\
& \leq C\left|\varrho_{0}, \mathbf{v}_{0}, \mathbf{H}_{0}\right|_{s}^{2}+C \int_{0}^{t} \sigma(1+\tau)^{2 \sigma-1}\left|\partial_{x} \partial_{\tau}^{j} \mathbf{H}\right|_{L^{2}}^{2} d \tau \\
& \quad+C \int_{0}^{t}(1+\tau)^{2 \sigma}\left|\left\langle\partial_{\tau}^{j} S_{3}, \partial_{\tau}^{j+1} \mathbf{H}\right\rangle\right| d \tau,
\end{aligned}
$$

for $2 j+1 \leq s$

iii) Due to Lemma 3.7, we have

$$
\begin{aligned}
& (1+t)^{2 \sigma}\left|\partial_{x}^{k+2} \partial_{t}^{j} \mathbf{H}(t)\right|_{L^{2}}^{2} \leq C(1+t)^{2 \sigma} \\
& \quad \times\left(\left|\partial_{t}^{j} \mathbf{H}\right|_{k}^{2}+\left|\partial_{t}^{j} S_{3}\right|_{k}^{2}+\left|\partial_{x} \partial_{t}^{j} \mathbf{H}\right|_{L^{2}}^{2}\right), \\
& \text { for } k+2 j+2 \leq s
\end{aligned}
$$

and

$$
\begin{aligned}
& \int_{0}^{t}(1+\tau)^{2 \sigma}\left|\partial_{x}^{k+2} \partial_{\tau}^{j} \mathbf{H}\right|_{L^{2}}^{2} \leq C \int_{0}^{t}(1+\tau)^{2 \sigma} \\
& \quad \times\left(\left|\partial_{\tau}^{j} \mathbf{H}\right|_{k}^{2}+\left|\partial_{\tau}^{j} S_{3}\right|_{k}^{2}+\left|\partial_{x} \partial_{\tau}^{j} \mathbf{H}\right|_{L^{2}}^{2}\right) d \tau,
\end{aligned}
$$

for $k+2 j+1 \leq s$.

By Cauchy inequality and $\sigma \geq \frac{1}{2}$, we have $(1+t)^{2 \sigma-1} \leq$ $C(\alpha)+\alpha(1+t)^{2 \sigma}, \forall \alpha>0$. This combining with (90)-(91), we get

$$
\begin{aligned}
& \sum_{k=0}^{\left[\frac{s}{2}\right]}\left\{(1+t)^{2 \sigma}\left|\partial_{t}^{k} \mathbf{H}(t)\right|_{L^{2}}^{2}+\int_{0}^{t}(1+\tau)^{2 \sigma}\left|\partial_{x} \partial_{\tau}^{k} \mathbf{H}\right|_{L^{2}}^{2} d \tau\right\} \\
& \quad+\sum_{j=0}^{\left[\frac{s-1}{2}\right]}\left\{(1+t)^{2 \sigma}\left|\partial_{x} \partial_{t}^{j} \mathbf{H}(t)\right|_{L^{2}}^{2}+\int_{0}^{t}(1+\tau)^{2 \sigma}\left|\partial_{\tau}^{j+1} \mathbf{H}\right|_{L^{2}}^{2} d \tau\right\} \\
& \leq C\left(\left|\varrho_{0}, \mathbf{v}_{0}, \mathbf{H}_{0}\right|_{s}^{2}+\int_{0}^{t}(1+\tau)^{2 \sigma-1}|\mathbf{H}|_{L^{2}}^{2} d \tau+\sum_{k=1}^{\left[\frac{s}{2}\right]} \int_{0}^{t}\left|\partial_{\tau}^{k} \mathbf{H}\right|_{L^{2}}^{2} d \tau\right. \\
& \quad+\sum_{j=0}^{\left[\frac{s-1}{2}\right]} \int_{0}^{t}\left|\partial_{x} \partial_{\tau}^{j} \mathbf{H}\right|_{L^{2}}^{2} d \tau+\sum_{k=0}^{\left[\frac{s}{2}\right]} \int_{0}^{t}(1+\tau)^{2 \sigma}\left|\left\langle\partial_{\tau}^{k} S_{3}, \partial_{\tau}^{k} \mathbf{H}\right\rangle\right| d \tau \\
& \left.\quad+\sum_{j=0}^{\left[\frac{s-1}{2}\right]} \int_{0}^{t}(1+\tau)^{2 \sigma}\left|\left\langle\partial_{\tau}^{j} S_{3}, \partial_{\tau}^{j+1} \mathbf{H}\right\rangle\right| d \tau\right) .
\end{aligned}
$$

for $2 j \leq s$. 
Multiplying (92) and (93) for $k=0,1$ with a sufficiently positive number $\beta$, and combining with (94), it yields

$$
\begin{aligned}
& \sum_{2 j+k \leq s, k \leq 3}\left\{(1+t)^{2 \sigma}\left|\partial_{t}^{j} \mathbf{H}(t)\right|_{k}^{2}+\sum_{2 j+k \leq s+1, k \leq 3} \int_{0}^{t}(1+\tau)^{2 \sigma}\left|\partial_{\tau}^{j} \mathbf{H}\right|_{k}^{2} d \tau\right\} \\
& \leq C\left(\left|\varrho_{0}, \mathbf{v}_{0}, \mathbf{H}_{0}\right|_{s}^{2}+\left.\int_{0}^{t}(1+\tau)^{2 \sigma-1}\left|\mathbf{H}_{L^{2}}^{2} d \tau+\sum_{k=1}^{\left[\frac{s}{2}\right]} \int_{0}^{t}\right| \partial_{\tau}^{k} \mathbf{H}\right|_{L^{2}} ^{2} d \tau\right. \\
& +\sum_{j=0}^{\left[\frac{s-1}{2}\right]} \int_{0}^{t}\left|\partial_{x} \partial_{\tau}^{j} \mathbf{H}_{L^{2}}^{2} d \tau+\sum_{k=0}^{\left[\frac{s}{2}\right]} \int_{0}^{t}(1+\tau)^{2 \sigma}\right|\left\langle\partial_{\tau}^{k} S_{3}, \partial_{\tau}^{k} \mathbf{H}\right\rangle \mid d \tau \\
& +\sum_{j=0}^{\left[\frac{s-1}{2}\right]} \int_{0}^{t}(1+\tau)^{2 \sigma}\left|\left\langle\partial_{\tau}^{j} S_{3}, \partial_{\tau}^{j+1} \mathbf{H}\right\rangle\right| d \tau+\sum_{2 j+k \leq s, 2 \leq k \leq 3}(1+t)^{2 \sigma}\left|\partial_{\tau}^{j} S_{3}\right|_{k-2}^{2} \\
& \left.+\sum_{2 j+k+1 \leq s, 2 \leq k \leq 3} \int_{0}^{t}(1+\tau)^{2 \sigma}\left|\partial_{\tau}^{j} S_{3}\right|_{k-2}^{2} d \tau\right) .
\end{aligned}
$$

For $k \geq 2$, the first term in the right-hand side of (92) and (93) can be absorbed by the left-hand side of (92) and (93) for $k=k-2$. Thus together with (95), we get (89).

In the proof of Subsubsection A priori estimates, we can obtain that $\mathcal{K}_{0}^{s}(t)^{2}+\mathcal{L}_{0}^{s}(t)^{2} \leq C\left|\varrho_{0}, \mathbf{v}_{0}, \mathbf{H}_{0}\right|_{s}^{2}$. Combining this with Proposition 3.4, we can derive from Lemma 3.13, for $\sigma=\frac{3}{4}-\varepsilon_{1}$ that

$$
\mathcal{K}_{\frac{3}{4}-\varepsilon_{1}}^{s}(t)^{2}+\mathcal{L}_{\frac{3}{4}-\varepsilon_{1}}^{s}(t)^{2} \leq C\left(\delta_{1}^{\prime}\right)+C \mathcal{N}_{\frac{3}{4}-\varepsilon_{1}}^{s}(t)^{2}
$$

here $C\left(\delta_{1}^{\prime}\right) \rightarrow 0$, as $\delta_{1}^{\prime} \rightarrow 0$. Hence it remains to estimate $\mathcal{N}_{\frac{3}{4}-\varepsilon_{1}}^{s}(t)$. We shall show that

$\mathcal{N}_{\frac{3}{4}-\varepsilon_{1}}^{s}(t)^{2} \leq \varepsilon \mathcal{L}_{\frac{3}{4}-\varepsilon_{1}}^{s}(t)^{2}+C(\varepsilon)\left\{\mathcal{K}_{\frac{3}{4}-\varepsilon_{1}}^{s}(t)^{4}+\mathcal{K}_{\frac{3}{4}-\varepsilon_{1}}^{s}(t)^{2} \mathcal{L}_{0}^{s}(t)^{2}\right\}$

Thus Proposition 3.5 follows. Since the other terms can be estimated in the similar way or we can see in (Kagei and Kobayashi 2005, here we only estimate $\int_{0}^{t}\left|\left\langle\partial^{k} \partial_{\tau}^{j}\left(\frac{1}{\rho} \operatorname{curl} \mathbf{H} \times \mathbf{H}\right), \partial^{k} \partial_{\tau}^{j} \mathbf{v}\right\rangle\right| d \tau$ with $k=s-2 j$ and $s \geq 3$ as

$$
\begin{aligned}
& \left|\left\langle l \partial^{k} \partial_{\tau}^{j}\left(\frac{1}{\rho} \operatorname{curl} \mathbf{H} \times \mathbf{H}\right), \partial^{k} \partial_{\tau}^{j} \mathbf{v}\right\rangle\right| \\
& =\left|\left\langle\partial^{s-1-2 j} \partial_{\tau}^{j}\left(\frac{1}{\rho} \operatorname{curl} \mathbf{H} \times \mathbf{H}\right), \partial^{s+1-2 j} \partial_{\tau}^{j} \mathbf{v}\right\rangle\right| \\
& \leq\left.\left|\partial^{s-1-2 j} \partial_{\tau}^{j}\left(\frac{1}{\rho} \operatorname{curl} \mathbf{H} \times \mathbf{H}\right)\right|_{L^{2}}|| \partial^{s+1-2 j} \partial_{\tau}^{j} \mathbf{v}\right|_{L^{2}} \\
& \leq C(\varepsilon)\left|\partial^{s-1-2 j} \partial_{\tau}^{j}\left(\frac{1}{\rho} \operatorname{curl} \mathbf{H} \times \mathbf{H}\right)\right|_{L^{2}}^{2}+\frac{\varepsilon}{2}\left|\partial^{s+1-2 j} \partial_{\tau}^{j} \mathbf{v}\right|_{L^{2}}^{2} .
\end{aligned}
$$

In virtue of (17) and its general form, and with the help of the smallness assumption in Proposition 3.4, we have i) While $j=0$,

$$
\begin{aligned}
\left|\partial^{s-1}\left(\frac{1}{\rho} \operatorname{curl} \mathbf{H} \times \mathbf{H}\right)\right|_{L^{2}}^{2} \leq & \left|\frac{1}{\rho}\right|_{L^{\infty}}^{2}|\mathbf{H}|_{L^{\infty}}^{2}\left|\partial_{x} \mathbf{H}\right|_{s-1}^{2} \\
& +\left|\frac{1}{\rho}\right|_{L^{\infty}}^{2}|\mathbf{H}|_{s-1}^{2}\left|\partial_{x} \mathbf{H}\right|_{L^{\infty}}^{2} \\
& +\left|\partial_{x}\left(\frac{1}{\rho}\right)\right|_{L^{\infty}}^{2}|\mathbf{H}|_{L^{\infty}}^{2}\left|\partial_{x} \mathbf{H}\right|_{s-2}^{2} \\
& +\left|\partial_{x}\left(\frac{1}{\rho}\right)\right|_{L^{\infty}}^{2}|\mathbf{H}|_{s-2}^{2}\left|\partial_{x} \mathbf{H}\right|_{L^{\infty}}^{2} \\
& +\left|\partial_{x}\left(\frac{1}{\rho}\right)\right|_{s-2}^{2}|\mathbf{H}|_{L^{\infty}}^{2}\left|\partial_{x} \mathbf{H}\right|_{L^{\infty}}^{2} \\
\leq & C|\varrho, \mathbf{H}|_{s-1}^{2}\left|\partial_{x} \mathbf{H}\right|_{s-1}^{2} .
\end{aligned}
$$

ii) While $1 \leq j \leq\left[\frac{s-1}{2}\right]$,

$$
\begin{aligned}
& \left|\partial^{s-1-2 j} \partial_{t}^{j}\left(\frac{1}{\rho} \operatorname{curl} \mathbf{H} \times \mathbf{H}\right)\right|_{L^{2}}^{2} \\
& \leq \sum_{1 \leq j_{1}+j_{2}+\cdots+j_{k} \leq j} \mid f\left(\rho ; j_{1}, j_{2}, \cdots, j_{k-2}\right)\left(\operatorname{sgn}\left(j_{1}\right) \partial_{t}^{j_{1}} \varrho\right) \cdots \\
& \quad \times\left(\operatorname{sgn}\left(j_{k-2}\right) \partial_{t}^{j_{k-2}} \varrho\right) \\
& \quad \times\left.\left(\operatorname{curl} \partial_{t}^{j_{k-1}} \mathbf{H} \times \partial_{t}^{j_{k}} \mathbf{H}\right)\right|_{s-1-2 j} ^{2} \\
& \leq C \sum_{j=1}^{\left[\frac{s-1}{2}\right]}\left|\partial_{t}^{j}(\varrho, \mathbf{H})\right|_{s-1-2 j}^{2} \sum_{l=1}^{\left[\frac{s-1}{2}\right]}\left|\partial_{x} \partial_{t}^{l} \mathbf{H}\right|_{s-1-2 l}^{2},
\end{aligned}
$$

where $f=\frac{c}{\rho^{m}}, c$ is a nonzero integer and $m=$ $m\left(j_{1}, j_{2}, \cdots, j_{k-2}\right)$ is some positive integer. And the last inequality in $i$ ) can be derived from the continuous equation $(19)_{1}$ and the smallness assumption of initial data. This together with the definition of $\mathcal{L}_{0}^{s}(t), \mathcal{L}_{\frac{3}{4}-\varepsilon_{1}}^{s}(t)$ and $\mathcal{K}_{\frac{3}{4}-\varepsilon_{1}}^{s}(t)$, it implies

$$
\begin{aligned}
& \int_{0}^{t}(1+\tau)^{2\left(\frac{3}{4}-\varepsilon_{1}\right) t}\left\langle\partial^{k} \partial_{\tau}^{j}\left(\frac{1}{\rho} \operatorname{curl} \mathbf{H} \times \mathbf{H}\right), \partial^{k} \partial_{\tau}^{j} \mathbf{v}\right\rangle d \tau \\
& \leq \varepsilon \mathcal{L}_{\frac{3}{4}-\varepsilon_{1}}^{s}(t)^{2}+C(\varepsilon) \mathcal{K}_{\frac{3}{4}-\varepsilon_{1}}^{s}(t)^{2} \mathcal{L}_{0}^{s}(t)^{2} .
\end{aligned}
$$

Now we shall finish the proof of the last part of Theorem 2 .

Proposition 3.6. Let $s \geq 4$. Under the assumptions of Proposition 3.5, if there exists a positive constants $\delta_{2}$ such that $\left|\varrho_{0}, \mathbf{v}_{0}, \mathbf{H}_{0}\right|_{s}+\left|\varrho_{0}, \mathbf{v}_{0}, \mathbf{H}_{0}\right|_{L^{1}} \leq \delta_{2}^{\prime}$, then we have

$$
\left|\partial_{x}(\varrho, \mathbf{v}, \mathbf{H})\right|_{2} \leq C \delta_{2}^{\prime}(1+t)^{-\left(\frac{5}{4}-\varepsilon_{2}\right)}, \forall 0<\varepsilon_{2}<\widehat{\varepsilon}
$$

for all $t \geq 0$, where $\widehat{\varepsilon} \leq \widetilde{\varepsilon}$ is some positive number. 
In order to prove Proposition 3.6, we first set

$$
\begin{gathered}
\tilde{M}_{\sigma}^{3}(t)=\sup _{0 \leq \tau \leq t}(1+\tau)^{\sigma}\left(\left.\partial_{x}^{2}(\varrho, \mathbf{v}, \mathbf{H})(\tau)\right|_{L^{2}}+\left|\partial_{\tau}(\varrho, \mathbf{v}, \mathbf{H})(\tau)\right|_{1}\right), \\
\tilde{\mathcal{K}}_{\sigma}^{s}=\left\{\sum_{k=3}^{s} M_{\sigma}^{k}+\sum_{j=1}^{\left[\frac{s}{2}\right]} \sup _{0 \leq \tau \leq t}(1+\tau)^{\frac{3}{4}-\varepsilon}\left|\partial_{\tau}^{j} \mathbf{v}(\tau)\right|_{s-2 j}\right. \\
\left.+\sum_{j=1}^{\left[\frac{s+1}{2}\right]} \sup _{0 \leq \tau \leq t}(1+\tau)^{\frac{3}{4}-\varepsilon}\left|\partial_{\tau}^{j} \varrho(\tau)\right|_{s+1-2 j}\right\}^{\frac{1}{2}} \\
\widetilde{\mathcal{L}}_{\sigma}^{s}=\left\{\begin{array}{c}
\int_{0}^{t}(1+\tau)^{2 \sigma}\left(\left|\partial_{x} \partial \mathbf{v}\right|_{s}+\left|\partial_{3} \mathbf{v}\right|_{s-2}^{2},\right. \\
+\left|\partial_{x}^{2} \varrho\right|_{s-2}+\left|\partial_{x} \partial_{\tau}(\varrho, \mathbf{v})\right|_{s-2}^{2} \\
\left.\quad+\sum_{j=2}^{\left.\frac{s+1}{2}\right]} \int_{0}^{t}(1+\tau)^{2 \sigma}\left|\partial_{\tau}^{j}(\mathbf{v}, \varrho)\right|_{s+1-2 j}\right) d \tau
\end{array}\right\}^{\frac{1}{2}},
\end{gathered}
$$

and

$$
\begin{aligned}
& \tilde{\mathcal{N}}_{\sigma}^{s}=\left\{\sup _{0 \leq \tau \leq t}(1+\tau)^{2 \sigma}\left|\partial_{x}^{2} S_{1}(\tau)\right|_{s-3}^{2}\right. \\
& +\sum_{j=1}^{\left[\frac{s-1}{2}\right]} \sup _{0 \leq \tau \leq t}(1+\tau)^{2 \sigma}\left|\partial_{\tau}^{j} S_{1}(\tau)\right|_{s-1-2 j}^{2} \\
& +\int_{0}^{t}(1+\tau)^{2 \sigma}\left(\left|\partial_{x}(\varrho \operatorname{divv})(\tau)\right|_{s-1}^{2}\right. \\
& \left.+\sum_{j=1}^{\left[\frac{s}{2}\right]}\left|\partial_{\tau}^{j}(\varrho \operatorname{divv})(\tau)\right|_{s-2 j}^{2}\right) d \tau \\
& +\int_{0}^{t}(1+\tau)^{2 \sigma}\left(\left|\partial_{x}^{2}(\mathbf{v} \cdot \nabla \varrho)(\tau)\right|_{s-3}^{2}\right. \\
& \left.+\sum_{j=1}^{\left[\frac{s-1}{2}\right]}\left|\partial_{\tau}^{j}(\mathbf{v} \cdot \nabla \varrho)(\tau)\right|_{s-1-2 j}^{2}\right) d \tau+(1+\tau)^{2 \sigma}\left|\partial_{x}\left(S_{2}, S_{3}\right)(\tau)\right|_{s-3}^{2} \\
& +\sum_{j=1}^{\left[\frac{s-2}{2}\right]} \sup _{0 \leq \tau \leq t}(1+\tau)^{2 \sigma}\left|\partial_{\tau}^{j}\left(S_{2}, S_{3}\right)(\tau)\right|_{s-2-2 j}^{2} \\
& +\int_{0}^{t}(1+\tau)^{2 \sigma}\left(\left|\partial_{x} S_{2}\right|_{s-2}^{2}+\sum_{j=1}^{\left[\frac{s-1}{2}\right]}\left|\partial_{\tau}^{j}\left(S_{2}, S_{3}\right)\right|_{s-1-2 j}^{2}\right) d \tau \\
& +\sum_{0 \leq k+2 j \leq s,(k, j) \neq(0,0)} \int_{0}^{t}(1+\tau)^{2 \sigma}\left|\left\langle\partial^{k} \partial_{\tau}^{j} S, \partial^{k} \partial_{\tau}^{j} V\right\rangle\right| d \tau \\
& +\sum_{0 \leq k+2 j \leq s-1,(k, j) \neq(0,0)} \int_{0}^{t}(1+\tau)^{2 \sigma}\left|\left\langle\partial^{k} \partial_{\tau}^{j} S, \partial^{k} \partial_{\tau}^{j+1} V\right\rangle\right| d \tau \\
& +\sum_{0 \leq k+l+2 j \leq s-1,(k, l, j) \neq(0,0,0)} \int_{0}^{t}(1+\tau)^{2 \sigma} \gamma \mu \mid\left\langle\partial^{k} \partial_{3}^{l+1} \partial_{\tau}^{j}\right. \\
& \left.\left.\times(\mathbf{v} \cdot \nabla \varrho), \partial^{k} \partial_{3}^{l+1} \partial_{\tau}^{j} \varrho\right\rangle \mid d \tau\right\}^{\frac{1}{2}} .
\end{aligned}
$$

As in the proof of Lemma 3.13 or we can see in (Kagei and Kobayashi 2005), the following inequality can be easily deduced:

Lemma 3.14. Let $s \geq 2$. Then under the assumptions of Proposition 3.5, the following inequalities hold uniformly in $t \geq 0$,

$$
\begin{aligned}
& \widetilde{\mathcal{K}}_{\frac{5}{4}-\varepsilon_{2}}^{s}(t)^{2}+{\widetilde{\mathcal{L}^{5}}}_{\frac{5}{4}-\varepsilon_{2}}^{s}(t)^{2} \\
& \leq C \\
& \quad C \mathcal{K}_{0}^{s}(t)^{2}+\mathcal{L}_{0}^{s}(t)^{2}+\tilde{\mathcal{N}}_{\frac{5}{4}-\varepsilon_{2}}^{s}(t)^{2} \\
& \left.\quad+\int_{0}^{t}(1+\tau)^{2\left(\frac{5}{4}-\varepsilon_{2}\right)-1}\left(\left|\partial_{x}(\varrho, \mathbf{v}, \mathbf{H})\right|_{L^{2}}^{2}+\left|\partial_{\tau}(\varrho, \mathbf{v}, \mathbf{H})\right|_{L^{2}}^{2}\right) d \tau\right\} .
\end{aligned}
$$

And we estimate $\tilde{\mathcal{N}}_{\frac{5}{4}-\varepsilon_{2}}^{s}(t)$ as:

Lemma 3.15. Let $s \geq 2$. Then under the assumptions of Proposition 3.5 , the following inequalities hold uniformly in $t \geq 0$,

$$
\begin{aligned}
\tilde{\mathcal{N}}_{\frac{5}{4}-\varepsilon_{2}}^{s}(t)^{2} \leq & C \mathcal{K}_{\frac{3}{4}-\varepsilon_{1}}^{s}(t)^{4}+\mathcal{K}_{\frac{3}{4}-\varepsilon_{1}}^{s}(t)^{2} \mathcal{L}_{\frac{3}{4}-\varepsilon_{1}}^{s}(t)^{2} \\
& +C\left\{M_{\infty}(t)+M_{\frac{5}{4}}^{1}(t)+\tilde{M}_{\frac{5}{4}}^{3}(t)+\tilde{\mathcal{K}}_{\frac{5}{4}-\varepsilon_{2}}^{s}(t)\right\} \\
& \times \mathcal{L}_{\frac{3}{4}-\varepsilon_{1}}^{s}(t)^{2} .
\end{aligned}
$$

Proof. Set $\tilde{\mathcal{N}}_{\frac{5}{4}-\varepsilon_{2}}^{s}(t)=J_{1}+J_{2}+\cdots+J_{10}$. Here we only consider $s \geq 4$ since the case while $2 \leq s<4$ can be deduced more easily. Thus we will estimate $J_{1}-J_{10}$ term by term.

$$
\begin{aligned}
J_{1}, J_{2} \leq & C \sup _{0 \leq \tau \leq t}(1+\tau)^{2\left(\frac{5}{4}-\varepsilon_{2}\right)}\left(\left|\partial_{x}^{3}(\varrho \mathbf{v})\right|_{s-3}^{2}+\sum_{j=1}^{\left[\frac{s-1}{2}\right]}\left|\partial_{\tau}^{j}(\varrho \mathbf{v})\right|_{s-2 j}^{2}\right) \\
\leq & C \sup _{0 \leq \tau \leq t}(1+\tau)^{2\left(\frac{5}{4}-\varepsilon_{2}\right)}\left(|\varrho, \mathbf{v}|_{L^{\infty}}^{2}|\varrho, \mathbf{v}|_{s}^{2}+\sum_{j=1}^{\left[\frac{s-1}{2}\right]}|\varrho, \mathbf{v}|_{L^{\infty}}^{2}\left|\partial_{\tau}^{j}(\varrho, \mathbf{v})\right|_{s-2 j}^{2}\right. \\
& \left.+\sum_{j=1}^{\left[\frac{s-1}{2}\right]} \sum_{k=1}^{j-1}\left|\partial_{\tau}^{k} \varrho, \partial_{\tau}^{j-k} \mathbf{v}\right|_{L^{\infty}}^{2}\left|\partial_{\tau}^{k} \varrho, \partial_{\tau}^{j-k} \mathbf{v}\right|_{s-2 j}^{2}\right) \\
\leq & C \sup _{0 \leq \tau \leq t}(1+\tau)^{2\left(\frac{5}{4}-\varepsilon_{2}\right)}\left(|\varrho, \mathbf{v}|_{s}^{4}+|\varrho, \mathbf{v}|_{s}^{2} \sum_{j=1}^{\left[\frac{s-1}{2}\right]}\left|\partial_{\tau}^{j}(\varrho, \mathbf{v})\right|_{s-2 j}^{2}\right. \\
& \left.+\sum_{k=1}^{\left[\frac{s-1}{2}\right]-1}\left|\partial_{\tau}^{k} \varrho\right|_{s-2 k-1}^{2} \sum_{l=1}^{\left[\frac{s-1}{2}\right]-1}\left|\partial_{\tau}^{l} \mathbf{v}\right|_{s-2 l-1}^{2}\right) \\
\leq & C \mathcal{K}_{\frac{3}{4}-\varepsilon_{1}}^{s}(t)^{4} .
\end{aligned}
$$

And this holds similarly for $J_{5}, J_{6}$. For the terms $J_{3}, J_{4}$ and $J_{7}$, we only estimate $\int_{0}^{t}(1+\tau)^{2\left(\frac{5}{4}-\varepsilon_{2}\right)} \mid\left.\partial_{x}(\varrho$ divv $)\right|_{s-1} ^{2} d \tau$ 
contained in $J_{3}$. The estimates of the other terms can arrive in the similar way.

$$
\begin{aligned}
& \int_{0}^{t}(1+\tau)^{2\left(\frac{5}{4}-\varepsilon_{2}\right)}\left|\partial_{x}(\varrho \operatorname{div} \mathbf{v})\right|_{s-1}^{2} d \tau \\
& \quad \leq C \int_{0}^{t}(1+\tau)^{2\left(\frac{5}{4}-\varepsilon_{2}\right)}|\varrho \operatorname{div} \mathbf{v}|_{s}^{2} d \tau \\
& \quad \leq C \int_{0}^{t}(1+\tau)^{2\left(\frac{5}{4}-\varepsilon_{2}\right)}\left(|\varrho|_{L^{\infty}}^{2}\left|\partial_{x} \mathbf{v}\right|_{s}^{2}+|\varrho|_{s}^{2}\left|\partial_{x} \mathbf{v}\right|_{L^{\infty}}^{2}\right) d \tau \\
& \quad \leq C \int_{0}^{t}(1+\tau)^{2\left(\frac{5}{4}-\varepsilon_{2}\right)}|\varrho|_{s}^{2}\left|\partial_{x} \mathbf{v}\right|_{s}^{2} d \tau \\
& \quad \leq C \mathcal{K}_{\frac{3}{4}-\varepsilon_{1}}^{s}(t)^{2} \mathcal{L}_{\frac{3}{4}-\varepsilon_{1}}^{s}(t)^{2} .
\end{aligned}
$$

For $J_{8}-J_{10}$, we only estimate the term $\sum_{1 \leq k \leq s-1}$ $\int_{0}^{t}(1+\tau)^{2\left(\frac{5}{4}-\varepsilon_{2}\right)}\left|\partial_{x}^{k} S_{3} \| \partial_{x}^{k} \partial_{\tau} \mathbf{H}\right| d \tau$ as

$$
\begin{aligned}
& \sum_{1 \leq k \leq s-1} \int_{0}^{t}(1+\tau)^{2\left(\frac{5}{4}-\varepsilon_{2}\right)}\left|\partial_{x}^{k} S_{3}\right|\left|\partial_{x}^{k} \partial_{\tau} \mathbf{H}\right| d \tau \\
& \leq C \sum_{1 \leq k \leq s-1} \int_{0}^{t}(1+\tau)^{2\left(\frac{5}{4}-\varepsilon_{2}\right)}\left|\partial_{x}^{k+1}(\mathbf{v} \times \mathbf{H})\right|\left|\partial_{x}^{k} \partial_{\tau} \mathbf{H}\right| d \tau \\
& \leq C \sum_{1 \leq k \leq s-1} \int_{0}^{t}(1+\tau)^{2\left(\frac{5}{4}-\varepsilon_{2}\right)}\left(|\mathbf{v}, \mathbf{H}|_{L^{\infty}}\left|\partial_{x}^{k+1}(\mathbf{v}, \mathbf{H})\right|_{L^{2}}\right. \\
& \quad+\left|\partial_{x}(\mathbf{v}, \mathbf{H})\right|_{L^{3}}\left|\partial_{x}^{k}(\mathbf{v}, \mathbf{H})\right|_{L^{6}} \\
& \left.\quad+\sum_{2 \leq j \leq k-1}\left|\partial_{x}^{j}(\mathbf{v}, \mathbf{H})\right|_{L^{6}}\left|\partial_{x}^{k+1-j}(\mathbf{v}, \mathbf{H})\right|_{L^{3}}\right)\left|\partial_{x}^{k} \partial_{\tau} \mathbf{H}\right|_{L^{2}} d \tau \\
& \leq C \sum_{1 \leq k \leq s-1} \int_{0}^{t}(1+\tau)^{2\left(\frac{5}{4}-\varepsilon_{2}\right)}\left(|\mathbf{v}, \mathbf{H}|_{L^{\infty}}+\left|\partial_{x}(\mathbf{v}, \mathbf{H})\right|_{L^{2}}\right. \\
& \left.\quad+\left|\partial_{x}^{2}(\mathbf{v}, \mathbf{H})\right|_{L^{2}}+\sum_{3 \leq j \leq k}\left|\partial_{x}^{j}(\mathbf{v}, \mathbf{H})\right|_{L^{2}}\right)\left|\partial_{x}^{k+1}(\mathbf{v}, \mathbf{H})\right|_{L^{2}}\left|\partial_{x}^{k} \partial_{\tau} \mathbf{H}\right|_{L^{2}} d \tau \\
& \leq C\left\{M_{\infty}(t)+M_{\frac{5}{4}}^{1}(t)+\widetilde{M}_{\frac{5}{4}}^{3}(t)+\widetilde{\mathcal{K}}_{\frac{5}{4}-\varepsilon_{2}}^{s}(t)\right\} \mathcal{L}_{\frac{3}{4}-\varepsilon_{1}}^{s}(t)^{2},
\end{aligned}
$$

which finished the proof of Lemma 3.15.

By virtue of Lemma 3.14 and Lemma 3.15, it remains to estimate $\widetilde{M}_{\frac{5}{4}-\varepsilon_{2}}^{3}(t)$ to finish the proof of Proposition 3.6.

Lemma 3.16. Let $s \geq 4$. Then under the assumptions of Lemma 3.13, the following inequalities hold uniformly in $t \geq 0$,

$\widetilde{M}_{\frac{5}{4}}^{3}(t)^{2} \leq C\left\{\mathcal{K}_{\frac{3}{4}-\varepsilon_{1}}^{s}(t)^{2}+M_{\frac{3}{4}}^{0}(t)^{2}+M_{\frac{5}{4}}^{1}(t)^{2}+M_{\infty}(t)^{2}\right\}$.

Proof. By Lemma $3.8 \mathrm{ii}$ ), we have

$$
\left|\partial_{x}^{l} \partial_{t} I_{0}(t)\right|_{L^{2}} \leq C t^{-\frac{5}{4}}\left\{\left|U_{0}\right|_{L^{1}}+\left|\varrho_{0}\right|_{2}+\left|\mathbf{v}_{0}\right|_{1}\right\}
$$

for $t \geq 1$ and $l=0,1$. Now we shall estimate $\partial_{x}^{l} \partial_{t} I_{1}(t)$. Since

$$
\begin{aligned}
\partial_{t} I_{1}(t) & =\left.E(t-\tau) F(U, \mathbf{H})(\tau)\right|_{t-1} ^{t}+\int_{t-1}^{t} E_{t}(t-\tau) F(U, \mathbf{H})(\tau) d \tau \\
& =\left.E(t-\tau) F(U, \mathbf{H})(\tau)\right|_{t-1} ^{t}-\int_{t-1}^{t} E_{\tau}(t-\tau) F(U, \mathbf{H})(\tau) d \tau \\
& =\int_{t-1}^{t} E(t-\tau) \partial_{\tau} F(U, \mathbf{H})(\tau) d \tau,
\end{aligned}
$$

then by Lemma 3.9 and Lemma 3.12, we have

$$
\begin{aligned}
\left|\partial_{x}^{l} \partial_{t} I_{1}(t)\right|_{L^{2}} & \leq C \int_{t-1}^{t}(t-\tau)^{-\frac{l}{2}}\left|\partial_{\tau} F(U, \mathbf{H})(\tau)\right|_{H^{1} \times L^{2}} d \tau \\
& \leq C \int_{t-1}^{t}(t-\tau)^{-\frac{l}{2}}\left\{\left|\partial_{t} S_{1}\right|_{1}+\left|\partial_{t} S_{2}\right|_{L^{2}}\right\} d \tau \\
& \leq C(1+t)^{-\frac{5}{4}}\left\{\mathcal{K}_{\frac{3}{4}-\varepsilon}^{4}(t)^{2}+M_{\infty}(t)^{2}+M_{\frac{5}{4}}^{1}(t)^{2}\right\} .
\end{aligned}
$$

Similarly, we can get

$\partial_{t} I_{2}(t)=E(1) F(U, \mathbf{H})(t-1)+\int_{0}^{t-1} \partial_{t} E(t-\tau) \partial_{\tau} F(U, \mathbf{H})(\tau) d \tau$.

Thus by Lemma 3.8, Lemma 3.11 and Lemma 3.12,

$$
\begin{aligned}
&\left|\partial_{x}^{l} \partial_{t} I_{2}(t)\right|_{L^{2}} \\
& \leq C|F(U, \mathbf{H})(t-1)|_{1} \\
&+C \int_{0}^{t-1}(t-\tau)^{-\frac{5}{4}}|F(U, \mathbf{H})(\tau)|_{L^{1}} \\
&+e^{-C_{0}(t-\tau)}|F(U, \mathbf{H})(\tau)|_{H^{1} \times L^{2}} d \tau \\
& \leq C \int_{t-1}^{t} \tau^{-\frac{1}{2}}\left\{\left|\partial_{t} S_{1}\right|_{1}+\left|\partial_{t} S_{2}\right|_{L^{2}}\right\} d \tau \\
& \leq C(1+t)^{-\frac{5}{4}}\left\{M_{\infty}(t)^{2}+M_{\frac{3}{4}}^{0}(t)^{2}+M_{\frac{5}{4}}^{1}(t)^{2}\right. \\
&\left.+\mathcal{K}_{\frac{3}{4}-\varepsilon_{1}}^{3}(t)^{2}+N(0, t)^{2}\right\} .
\end{aligned}
$$

It then follows that

$$
\begin{aligned}
\left|\partial_{t} U(t)\right|_{1} \leq C(1+t)^{-\frac{5}{4}} & \left\{M_{\infty}(t)^{2}+M_{\frac{3}{4}}^{0}(t)^{2}+M_{\frac{5}{4}}^{1}(t)^{2}\right. \\
& \left.+\mathcal{K}_{\frac{3}{4}-\varepsilon_{1}}^{4}(t)^{2}+N(0, t)^{2}\right\} .
\end{aligned}
$$

This together with the magnetic term which can be treated in the similar way, one has

$$
\begin{aligned}
\left|\partial_{t}(\varrho, \mathbf{v}, \mathbf{H})\right|_{1} \leq C(1+t)^{-\frac{5}{4}} & \left\{M_{\infty}(t)^{2}+M_{\frac{3}{4}}^{0}(t)^{2}+M_{\frac{5}{4}}^{1}(t)^{2}\right. \\
& \left.+\mathcal{K}_{\frac{3}{4}-\varepsilon_{1}}^{4}(t)^{2}+N(0, t)^{2}\right\} .
\end{aligned}
$$

Next we estimate $\left|\partial_{x}^{2} U(t)\right|_{L^{2}}$ and $\left|\partial \partial_{x}^{2} \mathbf{v}\right|_{L^{2}}$. For $k=1,2$, we see from Lemma 3.8 that

$$
\left|\partial^{k} \partial_{x} I_{0}(t)\right|_{L^{2}} \leq C t^{-\frac{7}{4}}\left\{\left|U_{0}\right|_{L^{1}}+\left|\varrho_{0}\right|_{k+1}+\left|\mathbf{v}_{0}\right|_{k}\right\} .
$$


And by Lemma 3.9 and Lemma 3.12,

$$
\begin{aligned}
\left|\partial^{k} \partial_{x} I_{1}(t)\right|_{L^{2}} \leq & C \int_{t-1}^{t}(t-\tau)^{-\frac{1}{2}}\left(\left|S_{1}\right|_{k+1}+\left|S_{2}\right|_{k}\right) d \tau \\
\leq & C\left\{M_{\infty}(t)^{2}+M_{\frac{5}{4}}^{1}(t)^{2}+\mathcal{K}_{\frac{3}{4}-\varepsilon_{1}}^{k+2}(t)^{2}\right\} \\
& \times \int_{t-1}^{t}(t-\tau)^{-\frac{1}{2}}(1+\tau)^{-\frac{3}{2}} d \tau \\
\leq & C(1+t)^{-\frac{3}{2}}\left\{M_{\infty}(t)^{2}+M_{\frac{5}{4}}^{1}(t)^{2}+\mathcal{K}_{\frac{3}{4}-\varepsilon_{1}}^{k+2}(t)^{2}\right\} .
\end{aligned}
$$

By Lemma 3.8 and Lemma 3.12,

$$
\begin{aligned}
\left|\partial^{k} \partial_{x} I_{2}(t)\right|_{L^{2}} & \\
\leq & C \int_{0}^{t-1}(t-\tau)^{-\frac{5}{4}}\left|\left(S_{1}, S_{2}\right)(\tau)\right|_{L^{1}} d \tau \\
& +C \int_{0}^{t-1} e^{-C_{0}(t-\tau)}\left(\left|S_{1}(\tau)\right|_{k+1}+\left|S_{2}(\tau)\right|_{k}\right) d \tau \\
\leq & C\left\{M_{\frac{3}{4}}^{0}(t)^{2}+M_{\frac{5}{4}}^{1}(t)^{2}+\mathcal{K}_{\frac{3}{4}-\varepsilon_{1}}^{k+2}(t)^{2}\right\} \\
& \times \int_{0}^{t-1}(t-\tau)^{-\frac{5}{4}}(1+\tau)^{-\left(\frac{7}{4}-\varepsilon_{1}\right)} d \tau \\
& +C\left\{M_{\infty}(t)^{2}+M_{\frac{5}{4}}^{1}(t)^{2}+\mathcal{K}_{\frac{3}{4}-\varepsilon_{1}}^{k+2}(t)^{2}\right\} \\
& \times \int_{0}^{t-1} e^{-C_{0}(t-\tau)}(1+\tau)^{-\frac{3}{2}} d \tau \\
\leq & C(1+t)^{-\frac{5}{4}}\left\{M_{\infty}(t)^{2}+M_{\frac{3}{4}}^{0}(t)^{2}+M_{\frac{5}{4}}^{1}(t)^{2}+\mathcal{K}_{\frac{3}{4}-\varepsilon_{1}}^{k+2}(t)^{2}\right\} .
\end{aligned}
$$

Combining these with the magnetic term which has the similar estimates, it yields

$$
\begin{aligned}
&\left|\partial^{k} \partial_{x}(\varrho, \mathbf{v}, \mathbf{H})\right|_{L^{2}} \leq C(1+t)^{-\frac{5}{4}}\left\{\delta_{1}^{\prime}+M_{\infty}(t)^{2}\right. \\
&\left.+M_{\frac{3}{4}}^{0}(t)^{2}+M_{\frac{5}{4}}^{1}(t)^{2}+\mathcal{K}_{\frac{3}{4}-\varepsilon_{1}}^{k+2}(t)^{2}\right\}
\end{aligned}
$$

for $k=1,2$.

Next, we consider $\partial_{3}^{2}(\varrho, \mathbf{v})$. From the equation $(19)_{2}$, we have

$$
\begin{aligned}
2 \mu \partial_{3}^{2} \mathbf{v}= & \mathbf{v}_{t}+\gamma \nabla \varrho-\mu(\partial \cdot \partial) \mathbf{v}-\mu \partial \operatorname{div} \mathbf{v} \\
& -\mu \partial_{3}\left(\partial_{1} \mathbf{v}^{1}+\partial_{2} \mathbf{v}^{2}\right)-S_{2} .
\end{aligned}
$$

Thus by (100) and (101), it follows that

$$
\begin{aligned}
\left|\partial^{l} \partial_{3}^{2} \mathbf{v}\right|_{L^{2}} \leq & C\left(\left|\partial^{l} \mathbf{v}_{t}\right|_{L^{2}}+\left|\partial^{l} \partial_{x} \varrho\right|_{L^{2}}+\left|\partial^{l+1} \partial_{x} \mathbf{v}\right|_{L^{2}}+\left|\partial^{l} S_{2}\right|_{L^{2}}\right) \\
\leq & C(1+t)^{-\frac{5}{4}}\left\{M_{\infty}(t)^{2}+M_{\frac{3}{4}}^{0}(t)^{2}+M_{\frac{5}{4}}^{1}(t)^{2}\right. \\
& \left.+\mathcal{K}_{\frac{3}{4}-\varepsilon_{1}}^{4}(t)^{2}+\delta_{1}^{\prime}\right\} .
\end{aligned}
$$

Similarly by $(19)_{1}-(19)_{2}$, we have

$$
\begin{aligned}
\partial_{3} \varrho_{t} & +\frac{\gamma^{2}}{2 \mu} \partial_{3} \varrho=\frac{\gamma}{2 \mu} \mathbf{v}_{t}^{3}-\frac{\gamma}{2} \partial_{3}\left(\partial_{1} \mathbf{v}^{1}+\partial_{2} \mathbf{v}^{2}\right) \\
& +\frac{\gamma}{2}(\partial \cdot \partial) \mathbf{v}^{3}+\partial_{3} S_{1}+\frac{\gamma}{2 \mu} S_{2} .
\end{aligned}
$$

Differentiating this in $x_{3}$, multiplying $\partial_{3}^{2} \varrho$ to the result equation, and integrating on $\mathbb{R}_{+}^{3}$, we have

$$
\frac{d}{d t}\left|\partial_{3} \varrho\right|_{L^{2}}+\frac{\gamma}{\mu}\left|\partial_{3} \varrho\right|_{L^{2}} \leq C\left(\left|\partial_{x} \mathbf{v}_{t}, \partial \partial_{x}^{2} \mathbf{v}, \partial_{x}^{2} S_{1}, \partial_{x} S_{2}\right|_{L^{2}}\right)
$$

Thus by Gronwall's inequality, and with the help of Lemma 3.12, and (87)-(89), we obtain

$$
\begin{aligned}
\left|\partial_{3}^{2} \varrho\right|_{L^{2}} & e^{-\frac{\gamma}{4 \mu}(t-1)}\left|\partial_{3}^{2} \varrho(1)\right|_{L^{2}}+C \int_{1}^{t} e^{-\frac{\gamma}{2 \mu}(t-\tau)} \\
& \times\left(\left|\partial_{x} \mathbf{v}_{t}, \partial \partial_{x}^{2} \mathbf{v}, \partial_{x}^{2} S_{1}, \partial_{x} S_{2}\right|_{L^{2}}\right) d \tau \\
\leq & C N(0, t) e^{-\frac{\gamma}{4 \mu}(t-1)} \\
& +C\left\{M_{\infty}(t)^{2}+M_{\frac{3}{4}}^{0}(t)^{2}+M_{\frac{5}{4}}^{1}(t)^{2}+\mathcal{K}_{\frac{3}{4}-\varepsilon_{1}}^{4}(t)^{2}+\delta_{1}^{\prime}\right\} \\
& \times \int_{1}^{t} e^{-\frac{\gamma}{2 \mu}(t-\tau)}(1+\tau)^{-\frac{5}{4}} d \tau \\
\leq & C(1+t)^{-\frac{5}{4}}\left\{M_{\infty}(t)^{2}+M_{\frac{3}{4}}^{0}(t)^{2}+M_{\frac{5}{4}}^{1}(t)^{2}\right. \\
& \left.+\mathcal{K}_{\frac{3}{4}-\varepsilon_{1}}^{4}(t)^{2}+\delta_{1}^{\prime}\right\},
\end{aligned}
$$

this together with (101) and (102), we get the estimate of $\left|\partial_{x}^{2}(\varrho, \mathbf{v})\right|_{L^{2}}$. Hence it remains to estimate $\left|\partial_{x}^{2} \mathbf{H}\right|_{L^{2}}$. By Lemma 2.4 and (100), we have

$$
\begin{aligned}
\left|\partial_{x}^{2} \mathbf{H}\right|_{L^{2}} \leq & C\left(\left|\mathbf{H}_{t}\right|_{L^{2}}+\left|S_{3}\right|_{L^{2}}+\left|\partial_{x} \mathbf{H}\right|_{L^{2}}\right) \\
\leq & C(1+t)^{-\frac{5}{4}}\left\{M_{\infty}(t)^{2}+M_{\frac{3}{4}}^{0}(t)^{2}+M_{\frac{5}{4}}^{1}(t)^{2}\right. \\
& \left.+\mathcal{K}_{\frac{3}{4}-\varepsilon_{1}}^{4}(t)^{2}+\eta_{1}^{2}\right\} .
\end{aligned}
$$

Combining this with the above estimates, Lemma 3.16 follows.

As in (103), we can deduce the decay rates of higher order derivatives for the magnetic field by Lemma 3.8, Lemma 3.16 and (100). Thus we state the result which is better than one in Proposition 3.6 in the following:

Proposition 3.7. Under the assumptions of Proposition 3.6, the solution $\mathbf{H}$ of (19) 3 satisfies that for all $t \geq 0$,

$$
\left|\partial_{x}^{3} \mathbf{H}(t)\right|_{L^{2}} \leq C(1+t)^{-\frac{5}{4}} .
$$


In view of the above established, the proof of Theorem 2 is complete.

\section{Competing interests}

The authors declare that they have no competing interests.

\section{Authors' contributions}

Author QC composed this paper, and others have revised it many times for publication. Both authors read and approved the final manuscript.

\section{Acknowledgements}

Q. Chen's research is supported in part by National Natural Science Foundation of China-NSAF (Nos. 11226174, 11301439). Z. Tan's research is supported in part by National Natural Science Foundation of China-NSAF (No. 11271305)

\section{Author details}

'School of Applied Mathematics, Xiamen University of Technology, Ligong Road, 361024 Xiamen, China. ${ }^{2}$ School of Mathematical Sciences, Xiamen University, Siming South Road, 361005 Xiamen, China.

Received: 3 March 2014 Accepted: 6 June 2014

Published online: 11 July 2014

\section{References}

Chen Q, Tan Z (2010) Global existence and convergence rates of smooth solutions for the compressible magnetohydrodynamic equations. Nonlinear Anal Theory, Methods Appl 72:4438-4451

Chen Q, Tan Z (2012) Global existence in critical spaces for the compressible magnetohydrodynamic equations. Kinet Relat Models 5:743-787

Chen GQ, Wang DH (2002) Global solutions of nonlinear magneto-hydrodynamics with large initial data. J Differential Equations 182:344-376

Chen GQ, Wang DH (2003) Existence and continuous dependence of large solutions for the magneto-hydrodynamics equations. Z Angew Math Phys 54:608-632

Cho Y, Choe HJ, Kim H (2004) Unique solvability of the initial boundary value problems for compressible viscous fluids. J Math Pures Appl 83:243-275

Ducomet B, Feireisl E (2006) The equations of magneto-hydrodynamics: on the interaction between matter and radiation in the evolution of gaseous stars. Commun Math Phys 266:595-629

Fan JS, Yu W (2009) Strong solution to the compressible magneto-hydrodynamic equations with vacuum. Nonlinear Anal Rea World Appl 10:392-409

Galdi GP, Bris CL, Lelievre T (1994) An introduction to the mathematical theory of the Navier-Stokes equations. Springer-Verlag, New York

Gerebeau JF, Bris CL, Lelievre T (2006) Mathematical methods for the magneto-hydrodynamics of liquid metals. Oxford University Press, Oxford

Kagei Y, Kobayashi T (2005) Asymptotic behavior of solutions of the compressible Navier-Stokes equations on the half space. Arch Ration Mech Anal 177:231-330

Kawashima S, Okada M (1982) Smooth global solutions for the one-dimensional equations in magneto-hydrodynamics. Proc Japan Acad Ser A Math Sci 58:384-387

Kobayashi T (2002) Some estimates of solutions for the equations of motion of compressible viscous fluid in an exterior domain in $\mathbb{R}^{3}$. J Differential Equations 184:587-619

Kobayashi T, Shibata Y (1999) Decay estimates of solutions for the equations of motion of compressible viscous and heat-conductive gases in an exterior domain in $\mathbb{R}^{3}$. Comm Math Phys 200:621-659

Matsumura A, Nishida T (1979) The initial value problem for the equations of motion of compressible viscous and heatconductive fluids. Proc Japan Acad Ser A 55:337-342

Matsumura A, Nishida T (1980) The initial value problem for the equations of motion of viscous and heat-conductive gases. J Math Kyoto Univ 20:67-104
Matsumura A, Nishida T (1983) Initial boundary value problems for the equations of motion of compressible viscous and heat-conductive fluids. Commun Math Phys 89:445-464

Ströhmer S (1990) About compressible viscous fluid flow in a bounded regione. Pacific J Math 143:359-375

Tan Z, Wang YJ (2009) Global existence and large-time behavior of weak solutions to the compressible magneto-hydrodynamic equations with coulomb force. Nonlinear Anal Theory, Methods Appl 71:5866-5884

Vol'pert Al, Hudjaev SI (1972) On the Cauchy problem for composite systems of nonlinear differential equations. Math USSR-Sb 16:517-544

doi:10.1186/s40068-014-0020-z

Cite this article as: Chen and Tan: Global existence and convergence rates for the smooth solutions to the compressible magnetohydrodynamic equations in the half space. Environmental Systems Research 2014 3:20.

\section{Submit your manuscript to a SpringerOpen ${ }^{\mathcal{O}}$ journal and benefit from:}

- Convenient online submission

- Rigorous peer review

- Immediate publication on acceptance

- Open access: articles freely available online

- High visibility within the field

- Retaining the copyright to your article

Submit your next manuscript at $>$ springeropen.com 